\title{
Chinese Herbal Medicine on Cardiovascular Diseases and the Mechanisms of Action
}

\author{
Cuiqing Liu ${ }^{1 *}$ and Yu Huang ${ }^{2 *}$ \\ ${ }^{1}$ Department of Preventive Medicine, Basic Medical College, Zhejiang Chinese Medical University, Hangzhou, China, \\ ${ }^{2}$ School of Biomedical Sciences, Institute of Vascular Medicine and Li Ka Shing Institute of Health Sciences, Chinese \\ University of Hong Kong, Hong Kong, China
}

Cardiovascular diseases are the principal cause of death worldwide. The potentially serious adverse effects of therapeutic drugs lead to growing awareness of the role of Chinese herbal medicine in the treatment of cardiovascular diseases. Chinese herbal medicine has been widely used in many countries especially in China from antiquity; however, the mechanisms by which herbal medicine acts in the prevention and treatment of cardiovascular diseases are far from clear. In this review, we briefly describe the characteristics of Chinese herbal medicine by comparing with western medicine. Then we summarize the formulae and herbs/natural products applied in the clinic and animal studies being sorted according to the specific cardiovascular diseases. Most importantly, we elaborate the existing investigations into mechanisms by which herbal compounds act at the cellular levels, including vascular smooth muscle cells, endothelial cells, cardiomyocytes and immune cells. Future research should focus on well-designed clinic trial, in-depth mechanic study, investigations on side effects of herbs and drug interactions. Studies on developing new agents with effectiveness and safety from traditional Chinese medicine is a promising way for prevention and treatment of patients with cardiovascular diseases.

Keywords: Chinese herbal medicine, hypertension, atherosclerosis, dyslipidemia, heart disease, cardiovascular diseases

Cardiovascular diseases, mainly including atherosclerosis, hypertension, cardiac hypertrophy, myocardial infarction and heart failure, are the principal cause of death worldwide. The increasing number of patients around the world suffering from CVDs indicates the need for innovative strategies for more effective prevention and treatment. Currently, western medicine, CHM and integrative medicine are the three major models of health care around the world. The western medicine has been considered as the mainstream medical treatment. For example, antithrombotic drugs combined with timely reperfusion therapy, coronary artery bypass grafting or precutaneous coronary intervention are widely applied to patients with CVDs. Although a widespread proof

Abbreviations: ANP, atrial natriuretic peptide; $\mathrm{BP}$, blood pressure; $\mathrm{BK}_{\mathrm{Ca}}$, channel $\mathrm{Ca}^{2+}$-activated $\mathrm{K}^{+}$channel; $\mathrm{CHM}$, Chinese herbal medicine; CVDs, cardiovascular diseases; ECM, extracellular matrix; (e)NOS, (endothelium) nitric oxide synthase; HDL-C, high-density lipoprotein cholesterol; HO, heme oxygenase; HUVEC, human umbilical vein endothelial cells; IL, interleukin; $\mathrm{K}_{\mathrm{ATP}}$, ATP-sensitive potassium; KRGE, Korean Red Ginseng extract; LDL, low-density lipoprotein; LDL-C, low-density lipoprotein cholesterol; NO, nitric oxide; PPAR $\alpha$, peroxisome proliferator-activated receptor-alpha; ROS, reactive oxygen species; Sal, salvianolic acid; SHR, spontaneous hypertension rats; SOD, superoxide dismutase; TC, total cholesterol; TG, triglyceride; VSMCs, vascular smooth muscle cells. 
showed these therapeutic regimes effective in reducing cardiovascular events, the potentially serious adverse effects are still key challenges. Thus it prompted the search for alternative and complementary therapies for better management of CVDs. CHM generally applies some natural plant products including dietary factors or herbal medicines. There is growing awareness of the role of herbs in the prevention of CVDs and the possibility of their use in treatment (Li et al., 2015). With the more successful clinic application of CHM in CVDs prevention and treatment, the effects of CHM have drawn greater attention, even in developed countries including the United States and Australia. In this review, we focus on both the clinic practices and experimental studies to summarize the application of plant products and mechanistic investigations.

\section{SEARCH STRATEGY}

To review all studies directly describing the application of CHM in CVDs, we used the keywords "cardiovascular" and "herbs" with limiting the search to title and/or abstract. The initial search generated 281 results. We then afterward selected the articles based on our aims. Since there are lots of formulae widely used in clinic in China, Guidelines for Diagnosis and Treatment of Common Internal Diseases in Chinese Medicine was referred too. A total of 123 articles and 1 Chinese Guideline was finally included in the review.

\section{COMPARISON BETWEEN CHM AND WESTERN MEDICINE}

Chinese herbal medicine is characterized by "Holistic regulation," in which the organism is considered as a whole. With the equilibrium of the human body as the guidelines, practitioners of CHM pay more attention to the diseased patients rather than the suffered diseases itself. Particular western medicine always has a strong effect on a specific disease with focusing on a specific physiological target and ignoring the specific characteristics of each patient.

"Syndrome Differentiation based treatment" is another feature of traditional Chinese medicine, which means diagnosis and treatment based on an overall analysis of the illness and practitioners timely modify formulae in accordance with the varying syndromes and clinical manifestations. Thus, it is an individualized treatment for different patients even with the same disease. Particular western medicine usually works against a specific pathological process of diseases rather than a patient.

Many CHM and foods originate from the same source and the feature of "Homology of medicine and food" reflects that CHM has few adverse effects. Traditionally, some foods are preferred to drugs for health care, and certain kinds of herbs are regarded as foods. Hawthorn fruit and garlic are such examples. However, due to the strong pertinence, western medicine will inevitably cause side effects. Although the efficacy of CHM is not as strong as chemicals on a pathological process of CVDs, the feature of individualized intervention with minimum adverse effects makes
CHM would be a good choice and will continue to make great contributions to the health of CVDs patients.

\section{APPLICATION OF CHM IN CVDs}

Many experimental studies indicate that a great many herbs have potential benefits for CVDs. Herbal plant-based formulations or drugs are pivotal to traditional practices and application of plant products as CHM treatment has been widely used in patients with hypertension, atherosclerosis, congestive heart failure, angina pectoris and other cardiovascular risk factors.

\section{Application of Plant Products in Hypertension}

Hypertension is a worldwide health problem with high morbidity and a major player in the onset of diseases such as atherosclerosis, stoke, peripheral artery disease, heart failure and coronary artery disease. Hypertension is defined as having a systolic BP of $\geq 140 \mathrm{mmHg}$ and a diastolic BP of $\geq 90 \mathrm{mmHg}$. Every 20/10 (systolic BP/diastolic BP) $\mathrm{mmHg}$ increase indicates a higher risk stage of hypertension, stage 1 (140-159/90-99 mmHg) and stage $2(\geq 160 / 100 \mathrm{mmHg}$ ) with the latter stage requiring immediate medical attention.

Notably, there are some formulae, many well known herbal products and their extracts or secondary metabolites of the herbs and spices exhibit antihypertensive effects. Interestingly, in a population-based database enrolling $984 \mathrm{CHM}$ users and 2434 non-CHM users with hypertension among type 2 diabetes patients, the CHM users were characterized with slightly longer duration time from diabetes to hypertension, suggesting that $\mathrm{CHM}$ may restrain hypertension pathogenesis among type 2 diabetes patients (Lin et al., 2015). Here, we present the most commonly used formulae and a comprehensive alphabetical list of plant products with evidence suggesting beneficial in hypertension therapy.

\section{Formulae}

Yiqi Huaju Formula and Bushen Qinggan Formula are regularly applied in clinical practice for treating hypertension. Yiqi Huaju Formula consists of Gastrodia elata, Uncaria, Eucommia bark, radix Scutellariae, and bitter butyl tea. In a study with 43 hypertensive patients coupled with metabolic syndrome, 12 weeks of additional use of Yiqi Huaju Formula (together with anti-hypertensive drugs) significantly decreased systolic BP and 24-h BP variability compared with the control group (anti-hypertensive drugs plus placebo), indicating that Yiqi Huaju treatment may be better than antihypertensive drugs alone (Chen et al., 2013). Bushen Qinggan Formula consists of Astragali Mongholici, Rhizoma Coptidis, Pollen Typhae, Rhizoma Alismatis, and Artemisiae Capillaries. A favorable effect of Bushen Qinggan Formula was observed on control of BP variability level after 8 weeks of treatment in a randomized controlled pilot clinical trial (150 patients with hypertension). This was accompanied with decreased endothelin and elevated nitric oxide (NO)/endothelin in circulation, indicating improved endothelial function ( $\mathrm{Wu}$ et al., 2014). In addition, according to the 
TABLE 1 | Formulae for hypertension based on syndrome differentiation.

\begin{tabular}{|c|c|c|c|c|}
\hline Symptom analysis & Therapeutic principle & Formulae & Ingredients & Chinese patent drug \\
\hline Gan Huo Shang Yan & Qing Gan Xie Huo & Long-Dan-Xie-Gan Tang & $\begin{array}{l}\text { Longdancao, Chaihu, Zexie, Cheqianzi, } \\
\text { Sheng Dihuang, Danggui, Zhizi, } \\
\text { Huangqin, Gancao. }\end{array}$ & $\begin{array}{l}\text { Xie Qing Wan, Dang Gui } \\
\text { Long Hui Wan }\end{array}$ \\
\hline Tan Shi Nei Zu & Hua Tan Qi Shi, He Wei Jiang Zhuo & $\begin{array}{l}\text { Ban-Xia-Bai-Shu-Tian-Ma } \\
\text { Tang }\end{array}$ & $\begin{array}{l}\text { Banxia, Baishu, Tianma, Chenpi, Fuling, } \\
\text { Gancao, Gouteng, Zhenzhumu, Yujin. }\end{array}$ & Xuan Yun Ning Tablet \\
\hline Yu Xue Nei Zu & Huo Xue Hua Yu & Tong-Qiao-Huo-Xue Tang & $\begin{array}{l}\text { Dilong, Danggui, Chuanqiong, Chishao, } \\
\text { Taoren, Honghua, Baizhi, Shichangpu, } \\
\text { Laocong, Quanxie }\end{array}$ & $\begin{array}{l}\text { Xin Mai Tong Tablet, Xin An } \\
\text { Ning Tablet }\end{array}$ \\
\hline Yin Xu Yang Kang & Ping Gan Qian Yang, Qing Huo Xi Feng & Tian-Ma-Gou-Teng Yin & $\begin{array}{l}\text { Tianma, Gouteng, Shijueming, Niuqi, } \\
\text { Duzhong, Sangjisheng, Huangqin, } \\
\text { Zhizi, Fushen, Yejiaoteng, Yimucao }\end{array}$ & $\begin{array}{l}\text { Qing Nao Jiang Ya Tablet, } \\
\text { Nao Li Qing Capsule }\end{array}$ \\
\hline Shen Jing Bu Zu & Zi Yang Gan Shen, Yi Jing Tian Sui & Zuo-Gui Wan & $\begin{array}{l}\text { Shu Dihuang, Shanzhuyu, Shanyao, } \\
\text { Guijia, Lujiaojiao, Gouqizi, Tusizi, Niuqi }\end{array}$ & $\begin{array}{l}\text { Jian Nao Bu Shen Wan, Yi } \\
\text { Ling Jing }\end{array}$ \\
\hline Qi Xue Liang Xu & Bu Yi Qi Xue, Tiao Yang Xin Pi & Gui-Pi Tang & $\begin{array}{l}\text { Dangshen, Baishu, Huangqi, Danggui, } \\
\text { Longyanrou, Dazao, Fushen, Yuanzhi, } \\
\text { Suanzaoren }\end{array}$ & $\mathrm{N} / \mathrm{A}$ \\
\hline Chong Ren Shi Tiao & Tiao She Chong Ren & Er-Xian Tang & $\begin{array}{l}\text { Xianmao, Xianlingpi, Danggui, Bajitian, } \\
\text { Huangbai, Zhimu, Baishao, Danshen, } \\
\text { Yimucao, Cheqianzi. }\end{array}$ & Lu Gui Bu Shen Capsule \\
\hline
\end{tabular}

Formulae should be modified according to specific syndromes and clinical manifestations.

Guidelines for Diagnosis and Treatment of Common Internal Diseases in Chinese Medicine, there are some frequently used formulae for hypertension in clinic which are based on syndrome differentiation (China Association of Chinese Medicine, 2008). The formulae are listed in Table 1.

\section{Natural Plant Products}

\section{Allium sativum L (Amaryllidaceae)}

Allium sativum $\mathrm{L}$ is also called garlic. It receives emerging interest from pharmacologists and health practitioners with its multi therapeutic effects, including hypotensive capacity, antiinflammatory, antioxidant, antibacterial and hypocholesteremic properties. Among the garlic's organo-sulfur constituents, allicin is the most responsible one. Fresh garlic directly releases allicin while chewing, whereas dried garlic preparations lack allicin but contain both allinin and allinase which could convert allinin to allicin. Evidence from many investigations and meta-analysis of randomized, controlled trials concluded that garlic supplements induce significant reduction in mean arterial pressure, drop in either systolic BP or diastolic BP with different extent. Interestingly, some studies indicate that aged garlic extract produces consistent lowering of BP compared to other forms of garlic (Blesken, 1992). However, more experiments are needed to confirm garlic's effect on hypertension since some studies come with no conclusive result (Shao et al., 2016). Despite having these multifarious effects, garlic also produces a few minor side effects, including abdominal swelling, heartburn, flatulence, and acid reflux et al.

\section{Andrographis paniculata (Acanthaceae)}

Andrographis paniculata is a plant that is commonly known as the King of Bitter. Several hypotensive labdane-type diterpenoid compounds, andrographolide, 14-deoxy- 11,12didehydroandrographolide and 14-deoxyandrographolide have been identified in Andrographis paniculata extracts. Animal studies show that treatment with its extracts decrease angiotensin converting enzyme and ROS activities in SHRs leading to decrease in BP (Zhang and Tan, 1996). Ex vivo studies demonstrate that the extracts reduce vascular resistance reflected by decreased coronary perfusion pressure in rat isolated hearts (Awang et al., 2012). However, no clinic trials have yet been conducted using the King of bitter.

\section{Apium graveolens L (Apiaceae)}

Apium graveolens $\mathrm{L}$ is also known as celery. The hypotensive effect of celery has been reported in in vivo animal studies. The extracts of celery reduce BP in deoxycorticosterone acetateinduced hypertensive rats or animal model of SHR (Moghadam et al., 2013). Importantly, extracts and constituents of celery have been reported to lower arterial pressure in humans, possibly by lowering levels of circulating catecholamines and decreasing vascular resistance (Houston, 2005).

\section{Bidens pilosa L (Compositae)}

Bidens pilosa $\mathrm{L}$ is also called Beggar's Tick. It is native to America and belongs to the family Compositae. Extracts of its leaves were able to prevent and attenuate high BP in different hypertensive rat models, both SHR and fructose-fed hypertensive rats (Steiner et al., 1996). In fructose-fed rats, 75 and $150 \mathrm{mg} / \mathrm{kg}$ of methanolic leaf extract of $B$. pilosa, showed both therapeutic and preventative effect on systolic BP (Steiner et al., 1996). However, clinical trials are required to determine the potential effect of this plant on hypertension.

\section{Camellia sinensis (Theaceae)}

Camellia sinensis, also known as tea, is most frequently consumed and only second to water. Its cardiovascular protective effect is well known worldwide. Catechins, the major 
flavonoids in tea, include (-)- epicatechin, (-)-epicatechin3-gallate, (-)- epigallocatechin, and (-)-epigallocatechin-3gallate. (-)-epigallocatechin-3-gallate constitutes the primary component of tea's total catechins. Although some trials come to conclusion of no change in BP subsequent to drinking tea, lots of studies suggest that tea consumption reduces both systolic BP and diastolic BP by $\sim 2 \mathrm{mmHg}$ each. Interestingly, the green tea evokes a more powerful hypotensive effect compared to black tea.

\section{Coptis chinensis (Ranunculaceae)}

It's commonly known as goldthread and widely used in Chinese folk medicine. The goldthread and its main component berberine, have the ability to lower BP. Indeed, a recent metaanalysis of twenty-seven randomized controlled trials involving 2569 patients reported that berberine can cause a significant hypotensive effect (Lan et al., 2015). In addition, this metaanalysis concluded that combined with an oral hypotensor, berberine can significantly reduce BP more than the hypotensor alone can do. The magnitude of the decrease was at an average of 4.91 and $2 \mathrm{mmHg}$ for systolic BP and diastolic BP, respectively (Lan et al., 2015).

\section{Crataegus spp. (Rosaceae)}

It is also known as Hawthorn or Shanzha. The plants are shrubs that belong to a genus comprising almost 300 species and have been used in traditional medicine for treatment of CVDs since the seventeenth century. Hawthorn is used to dissipate food accumulation, to improve blood circulation, and to disperse blood stasis. The antihypertensive actions should be credited to the plant's multiple components: flavonoids (hyperoside, quercetin, rutin, and vitexin) and oligomeric proanthocyanidins (OPCs, epicatechin, procyanidin, and procyanidin B-2). Modest decreases in BP have been observed in a few human-based studies with a demographic of hypertensive patients (Walker et al., 2002; Tassell et al., 2010). In a randomized, double-blind, placebocontrolled study where mildly hypertensive subjects were treated with $500 \mathrm{mg}$ of hawthorn extract for 10 weeks, a promising tendency for a reduction in diastolic BP was reported (Walker et al., 2002). In another clinic study, administration of hydroalcoholic extracts of hawthorn flowers to hypertensive patients (age range 40-60 years) for 3 months induced a decrease in both systolic BP and diastolic BP by around 13 and $8 \mathrm{mmHg}$, respectively (Asgary et al., 2004).

\section{Crocus sativus L (Iridaceae)}

Crocus sativus L, also known as Saffron, a plant indigenous to Southwest Asia, Spain, Greece, and Morocco, is a stemless herb. Saffron's main components include crocin, picrocrocin, safranal, and crocetin. Several reports support the use of saffron for antihypertensive benefits. A clinical study reports that $400 \mathrm{mg}$ of Saffron tablets administered for 7 days are able to significantly reduce the systolic $\mathrm{BP}$ and mean arterial pressure in healthy humans by 11 and $5 \mathrm{mmHg}$, respectively (Modaghegh et al., 2008). Six weeks old stroke-prone SHRs are given crocetin for 3 weeks and this treatment significantly moderates the increase in systolic BP observed with age (Higashino et al., 2014). Saffron also demonstrates vasorelaxant activities in different animal models, including male Sprague-Dawley rats (Fatehi et al., 2003), strokeprone SHRs (Higashino et al., 2014), deoxycorticosterone acetatesalt induced hypertensive male Wistar rats (Imenshahidi et al., 2010) but not normotensive rats (Imenshahidi et al., 2015).

\section{Hibiscus sabdariffa L (Malvaceae)}

Also known as Roselle, Hibiscus sabdariffa is widely used for hypertension, fever, and other diseases in folk medicine. Different parts of this plant (buds, calyx, flowers, leaves, and petalsfresh or dried) are used for health purposes and as refreshing beverages, food items (jams, preserves), or lotions. Hibiscus's effects of hypotension have been extensively investigated in both animal and human studies (McKay et al., 2010; Ojeda et al., 2010; Inuwa et al., 2012). The blood lowering effects were notable subsequent to treatment with dried extract of Roselle calyx for 4 weeks in patients with stage 1 or 2 hypertension (Herrera-Arellano et al., 2007). In addition, consuming Roselle's tea (240 $\mathrm{ml}$-three times a day for 6 weeks) significantly reduces systolic BP, diastolic BP, and mean arterial pressure by 7.2, 3.1 , and $4.5 \mathrm{mmHg}$, respectively in mild and pre-hypertensive patients (65 subjects, age range 30-70 years old) (McKay et al., 2010), supporting Hibiscus' therapeutic role in ameliorating hypertension.

\section{Hippophae rhamnoides L (Elaegnaceae)}

Hippophae rhamnoides L is also called Saji or Sea buckthorn and its dry fruits are used in China as an herbal medicine. Analysis showed that the powder made of dry hippophae fruits contains the vitamins $\mathrm{C}, \mathrm{B} 1, \mathrm{~B} 2$ and $\mathrm{E}$, provitamin $\mathrm{A}$, rutin, serotonin, cytosterol, selenium and zinc. The stroke-prone SHR were fed ad libitum with blocks of rat chow supplemented with Hippophae powder and the effect of lowering BP was examined. The mean arterial BP and heart rate were significantly decreased by the Hippophae treatment (Koyama et al., 2009), which is in accord with previous studies showing that Hippophae extracts decreased intracellular $\mathrm{Ca}^{2+}$ in cultured VSMCs (Zhu et al., 2005).

\section{Nigella sativa L (Ranunculaceae)}

Nigella sativa L, also known as Black Cumin or Habbatul barakah (seed of blessing), has been used in the kitchens of Europe, the Middle East, Africa, and Asia for centuries. Thymoquinone, one of the most abundant and bioactive components, has been identified as the major element in its healing effects. Similar to other herbs, $N$. sativa and its constituents have been shown to reduce BP in humans and different animal models of hypertension. $N$. sativa's seed extract administrated orally (either 100 or $200 \mathrm{mg}$, two times per $24 \mathrm{~h}$ for 8 weeks) to mild hypertensive male patients recorded a dose-dependent fall in both systolic BP and diastolic BP (Ried, 2016). Again, in a randomized, placebo-controlled, double-blind study with 70 healthy subjects, $N$. sativa oil caused a significant decrease of $10.6 \mathrm{mmHg}$ in systolic BP and $9.6 \mathrm{mmHg}$ in diastolic BP (Fallah Huseini et al., 2013).

\section{Panax ginseng C.A. Mey (Araliaceae)}

Panax ginseng, also called Panax or Renshen, has been used in folk medicine for several centuries. Ginseng is prepared 
and administered in various forms, either as a solid: tablets, capsules, dried roots; or as a liquid: oil, extracts or tea. There are four most common species of Ginseng, including $P$. ginseng (Asian or Korean Ginseng), P. quinquefolius (American Ginseng), P. japonicas (Japanese Ginseng) and $P$. notoginseng (Chinese Ginseng) are the four most common species of ginseng. Heterogeneous triterpenoid saponins and steroid glycosides or ginsenosides (or panaxosides) are the active principle components of ginseng. Several clinical trials have been conducted to assess the efficacy of ginseng in BP regulation. Both Asian/Korean Ginseng extract and American Ginseng caused a significant decrease in systolic BP and diastolic BP in hypertensive patients (Mucalo et al., 2013; Rhee et al., 2014). The mode of action may be related with antagonizing a calcium ion channel in vascular tissues, which may result in lowering BP. However, conflicting reports of elevated BP also exist (Jang et al., 2011; Kim, 2012). For example, studies have shown that low doses of ginseng raise $\mathrm{BP}$, while higher concentrations are hypotensive (Jang et al., 2011), which need more experiments to clarify the differentiation.

\section{Pueraria lobata (Willd) ohwi (Leguminosae)}

It is also known as Kudzu root or Gegen. Kudzu root has traditionally been used in Chinese medicine for treating CVDs and Type II diabetes. It is a rich source of polyphenolic compounds, including isoflavones, isoflavonoid glycosides, coumarins, puerarols, but-2-enolides and their derivatives. Puerarols attract more attention to investigate the direct effect on BP. According to Wong's review, the anti-hypertensive effect of puerarin was shown as early as 1980 s evidenced by reduced BP, heart rate and plasma rennin activity in SHRs and this effect may be exerted through blocking the effect of $\beta$-adrenergic receptors (Wong et al., 2011). Verapamil is a positive control inhibiting Angiotensin II type I receptor and angiotensin-converting enzyme 2 in SHRs. Similar to it, puerarin treatment significantly repressed the mRNA levels of Angiotensin II type I receptor and angiotensin-converting enzyme 2 in cardiac tissues (Ye et al., 2008). Further interdisciplinary collaboration to bridge the gap between traditional medicine and modern biomedical medicine is needed for the development of Kudzu root as an effective medicine for the management of hypertension and other CVDs.

\section{Salviae miltiorrhizae (Lamiaceae)}

It is also known as Danshen, or red/Chinese sage. Danshen is one of the oldest and most frequently consumed Chinese traditional herbs and is commonly used for the treatment of CVDs. Sal A, Sal B, Danshensu, and tanshinones are its most effective ingredients. A combination treatment of Danshen and Gegen was shown to lower BP in SHRs and to induce relaxation of several kinds of arteries including porcine coronary arteries, rat aorta, and basilar arteries (Al Disi et al., 2015). Apart from its vasodilatory capacity, Danshen expresses additional antihypertensive parameters such as antioxidative, anti-proliferative, and anti-inflammatory activities which will be discussed in more details in Section "Mechanisms of Plant Products in the Attenuation of CVDs.”

\section{Scutellaria baicalensis Georgi (Lamiaceae)}

The dried roots of $S$. baicalensis, also known as Huangqin in China, have been widely employed in traditional CHM as popular antibacterial and antiviral agents. Baicalein and its glycoside, baicalin, are the major ingredients responsible for its beneficial effects. Its BP lowering effect in dog and rat was reported as early as 1980's (Huang et al., 2005). However, the baicalein exerts a complex effect on the agonist contracted rat mesenteric arteries. Within a relative low concentration range $(0.3-10 \mu \mathrm{M})$, baicalein potentiates vasoconstricting responses to phenylephrine, U46619, or to elevated extracellular potassium in endothelium-intact artery rings (Chen et al., 1999; Tsang et al., 2000), whilst it produces relaxations at concentrations greater than $10 \mu \mathrm{M}$ (Chen et al., 1999). Since the increased vessel contraction is absent upon removal of the endothelium while the relaxant effect of baicalein remains (Chen et al., 1999), endothelium-derived vasoactive factors may be involved in the bidirectional effect on vascular function. To be specific, baicalein and baicalin increase the evoked contractile responses likely through inhibition of NO production and/or release in the endothelium (Huang et al., 2000, 2002; Tsang et al., 2000). The mechanisms by which baicalein lowers BP will be discussed in Section "Regulating Calcium Levels in PKA/PKG/PKC-Dependent Way."

\section{Application of Plant Products in Atherosclerosis and Dyslipidemia}

Atherosclerosis, one of the primary causes of CVDs, is a vascular disease that occurs at susceptible sites in major arteries. It is an inflammatory process and ultimately causes stenosis or thrombosis with potentially lethal distal ischemia. The main elements involved in the complex pathogenesis of atherosclerosis include hyperlipidemia, endothelial injury, LDL subendothelial retention and oxidation, monocyte migration and activation, VSMC migration and proliferation, foam cell formation, apoptosis and efferocytosis and unresolved inflammation. Among them, dyslipidemia is the primary independent risk factors, which is characterized by elevated level of total cholesterol (TC), triglyceride (TG), LDL-C and by lowered level of high-density lipoprotein cholesterol (HDL-C) in serum.

The description of the clinical manifestations and treatment of atherosclerosis can be found in the classic traditional Chinese medicine book Inner Canon of yellow Emperor, as early as 500BC. Atherosclerosis, dyslipidemia and its resulting heart disease have been treated with numerous herbal remedies for centuries. Then, we present a comprehensive alphabetical list of plant products which are most commonly used to attenuate atherosclerosis and lower hyperlipidemia.

\section{Allium fistulosum L (Amaryllidaceae)}

Allium fistulosum L is widely cultivated in Southern China. Treatment with Fistular onion stalk, the derivant from A. fistulosum., induces a significant reduction in the average lesion area of atherosclerosis and preservation of the vascular wall and immune cell infiltration. The extract also reduces 
the levels of the inflammatory cytokines IL-1 $\beta$, IL-6, MCP1 and $\mathrm{TNF} \alpha$ and downregulated the local activity of the renninangiotensin-aldosterone system in the aortic tissue. In addition, extract treatment inhibits several local inflammatory signaling pathways by preventing its activation, including phosphorylation of the NFKB, Janus kinase/signal transducers and activators of transcription and mitogen-activated protein kinase pathways (He B. et al., 2014). These data indicate that fistular onion stalk extract may be useful for the attenuation of atherosclerosis, and the mechanism includes the regulation of the local inflammatory responses.

\section{Allium sativum L (Amaryllidaceae)}

In addition to the anti-hypertensive effect, there is also wide spread belief among general public that Allium sativum $\mathrm{L}$ (garlic) has beneficial effects on dyslipidemia in patients. Seventy type 2 diabetes patients with newly diagnosed dyslipidemia were divided into two groups and were given tablet garlic $300 \mathrm{mg}$ (containing $1.3 \%$ allicin) twice daily and identical placebo tablets respectively. 12-week of garlic treatment induces a significant reduction in TC and LDL-C, a significant increase in HDL-C (Ashraf et al., 2005). In a double-blind, crossover study of moderately hypercholesterolemic men, treatment with $7.2 \mathrm{~g}$ aged garlic extract induced a maximal decrease of $6.1 \%$ in serum TC levels and $4.6 \%$ in LDL cholesterol levels (Steiner et al., 1996). These effects were Although one recent meta-analysis concluded that garlic decreases TC to a modest extent, an effect driven mostly by the modest decreases in TG, with no appreciable effect on LDL or HDL cholesterol (Reinhart et al., 2009), many studies suggest garlic to be effective in improving lipid metabolism (Ried, 2016). Since some meta-analysis has been based on trials with inadequate study designs, methodological deficiencies, the effect of garlic on atherosclerosis and dyslipidemia awaits further investigation.

\section{Astragalus propinquus (Fabaceae)}

The dried root of Astragalus propinquus, Radix Astragali, is also known as Huangqi. Polysaccharides, flavonoids, and sponins are the main active components of membranous milkvetch root. Studies have shown that Huangqi extract significantly decreased the area of atherosclerosis plaques $(17.24 \% \pm 4.22 \%$ vs. $49.87 \% \pm 9.37 \%, P<0.01)$ and level of serum oxLDL $(5.2 \pm 6.1 \mu \mathrm{g} / \mathrm{ml}$ vs. $15.8 \pm 5.4 \mu \mathrm{g} / \mathrm{ml}, P<0.01)$ in $\mathrm{ApoE}^{-/-}$ mice (You et al., 2012). Effects of its extract and components on lipid profile are also summarized in a review, in which the serum of TG, TC, and LDL-C levels were reduced by both Astragalus mongholicus extracts treatment at 0.4 and $0.8 \%$ for 5 weeks and polysaccharides from Astragalus administered at an oral dosage of $40 \mathrm{mg}, 100 \mathrm{mg} / \mathrm{kg} /$ day in hyperlipidemia rats for 40 days (Guo et al., 2014). Accordingly, the HDL-C levels were increased by these treatments (Guo et al., 2014).

\section{Coptis chinensis Franch. (Ranunculaceae)}

Rhizoma Coptidis, also known as Huanglian, is derived from the dried root and rhizome of Coptis chinensis Franch., Coptis deltoidea C. Y. Cheng et Hsiao, and Coptis teeta Wall. Its main components include lignans and alkaloid, in which berberine is the active component for lipid lowering. Because of berberine's active effect for lipid lowering, most of the studies focus on this component. Studies have shown that berberine administration significantly decreased the serum TG, TC, LDL-C, when taken orally or injected intraperitoneally (Zhou et al., 2008; Chang et al., 2012). In addition, Caucasian obese human subjects were orally given $500 \mathrm{mg}$ berberine, three times a day for 12 weeks. The blood lipid was significantly reduced and TG, TC were decreased by 23 and $12.2 \%$, respectively ( $\mathrm{Hu}$ et al., 2012). In a meta-analysis, berberine induced a significant reduction in these biomarkers in 874 participants in11 randomized controlled trials (Ma et al., 2013).

\section{Crataegus spp. (Rosaceae)}

Known as hawthorn, its hypolipidemia effect has been investigated widely in animal studies. In high-fat diets fed mice, the oral administration of the whole extract at a dosage of $250 \mathrm{mg} / \mathrm{kg} /$ day for 7 days induced blood lipid decrement (Niu et al., 2011). Then, the specific effects of aqueous and ethanol extracts of hawthorn on lipid profiles were compared. In a high-fat emulsion fed mice, both ethanol and aqueous extracts possessed hypolipidemia activities and the ethanol extract exhibited more favorable effects than the aqueous extract (Shao et al., 2016). This lipid lowering effect of hawthorn mostly contributes to inhibition of the progression of atherosclerosis which was evidenced by the significantly inhibited pathological changes and reduced intimamedia thickness in the arteries (Zhang et al., 2013). In-depth exploration demonstrated that the lipid-lowering action may be due to the anti-inflammation activities, the upregulation of PPAR $\alpha$ to facilitate $\beta$-oxidation-related enzymes in liver leading to lipid degradation, enhanced expression of hepatic LDL receptors resulting in a greater influx of plasma cholesterol into the liver, and the suppressed cholesterol biosynthesis and increased degradation of cholesterol to bile acids (Niu et al., 2011; Walden and Tomlinson, 2011; Zhang et al., 2013; Shao et al., 2016). The hypolipidemic effects of hawthorn in clinic remain further study.

\section{Epimedium brevicornum Maxim (Berberidaceae)}

Epimedium brevicornum Maxim, an important traditional CHM, has been used widely for 1000 s of years in China, Korea, and Japan. Icariin, a flavonoid isolated from Epimedium brevicornum Maxim, is considered as the main pharmacological active constituent. In the high cholesterol diet-induced atherosclerosis rats, the levels of blood lipids including TC, TG, LDL-C, and malonaldehyde were significantly increased, while HDL-C and SOD were significantly decreased. Icariin succeeded in improving these biochemical parameters toward the normal values ( $\mathrm{Hu}$ et al., 2016).

\section{Fallopia multiflora Thunb. (Polygonaceae)}

Its dried root tuber is Radix Polygoni Multiflori, also known as Heshouwu. Raw and prepared pharmaceutical forms are 
frequently used. Guo et al. (2014) reviewed the hypolipidemic effect of Heshouwu from some Chinese literature which demonstrated that the total extract of the Radix Polygoni Multiflori significantly reduced the serum levels of TC, TG and LDL-C in hyperlipidemic rats when administered at an oral dosage of $12 \mathrm{mg}$ and $24 \mathrm{mg} / \mathrm{kg} /$ day for 4 weeks. Similarly, these lipid profiles in the hyperlipidemic rats were lowered by the treatment of the ethlacetate extracting fraction and stilbene glycoside from the tube of Radix Polygonum Multiflorum too (Guo et al., 2014).

\section{Fermentum Rubrum (Aspergillaceae)}

Fermentum Rubrum, popularly known asred yeast rice, is the fermented product of Monascus purpureus on rice. It is composed of 13 kinds of natural statins, unsaturated fatty acids, ergosterol, amino acids, flavonoids, alkaloid, trace element, and so forth. Some clinic studies have demonstrated the lipid lowering effects of the red yeast rice. A twice daily dose of red yeast rice at $600 \mathrm{mg}$ for 8 weeks was found to reduce LDL-C by $27.7 \%$, TG by $21.5 \%$, and TC by $15.8 \%$ (Lin et al., 2005). Similar effects were observed in 72 patients with idiopathic persistent nephritic syndrome with secondary dyslipidemia (Gheith et al., 2008, 2009). Xuezhikang capsule is the extract of red yeast rice. Scholars in China made a systematic review on the clinical randomized controlled trials for hyperlipidemia treatment with Xuezhikang, which included a total of 6520 participants in 22 randomized trials. It was concluded that Xuezhikang remarkably lowered TC, TG, and LDL-C compared with the inositol nicotinate (Fallah Huseini et al., 2013).

\section{Olea europaea L. (Oleaceae)}

The beneficial effects of Olea europaeal L., especially olive leaf extract are known since antiquity, with numerous records confirming its therapeutic use. According to the European Pharmacopeia, the most abundant substances in standardized dry leaf extracts are oleuropein, hydroxytyrosol, caffeic acid, tyrosol, apigenin, and verbascoside, with oleuropein being the major component of olive leaf extract. Olive leaf extract treatment reduces the atherosclerostic lesions size and the thickness of intima in atherosclerotic rabbits, accompanied with decreased levels of atherosclerotic markers, including serum TG, TC, LDL, HDL, and malonaldehyde with a parallel downregulation of MCP1, vascular cell adhesion molecule1, NFkB and TNFo (Wang et al., 2008). Consistent with it, another animal study showed that a 1-month intake of hydroxytyrosol by rabbits consuming an atherogenic diet results in a reduced size of atherosclerotic lesions compared to control animals (Gonzalez-Santiago et al., 2006). However, the results originating from in vivo models are divergent. The intake of hydroxytyrosol in an in vivo apo-E knockout mice model showed that this compound, when administered for 10 weeks, leads to atherosclerotic lesions associated with the activation of monocytes and modification of the blood lipid profile (Acin et al., 2006). These studies indicate that under certain conditions the phenol could be rather harmful than cytoprotective.

\section{Panax ginseng C.A. Mey (Araliaceae)}

The resources and components have been discussed in Section "Application of Plant Products in Hypertension." A serious of studies has demonstrated the hypolipidemic effects of its components. For example, the levels of plasma TC, TG, and LDL-c in C57/BL-ApoE gene knockout hyperlipidemia rats were reduced by intragastric administration of ginseng saponins at an oral dosage of $2 \mathrm{mg} / \mathrm{kg} /$ day for 90 days. Ginseng saponin is divided into $\mathrm{Rb} 1, \mathrm{Rb} 2, \mathrm{RC}, \mathrm{Rd}, \mathrm{Re}$, and $\mathrm{Rl}$. Ginseng saponin $\mathrm{Rb}$ administered at an oral dosage of $50-200 \mathrm{mg} / \mathrm{kg} /$ day in hyperlipidemia rat for 12 days significantly reduce the TG, TC, and LDL-c levels in serum and liver (Yue et al., 2012). Ginseng radix can be metabolized into compound $\mathrm{k}$ in small intestinal. In vitro, the metabolites significantly activate the AMP-activated protein kinase (AMPK) to affect the lipid metabolism in insulinresistant HepG2 human hepatoma cells (Kim et al., 2009).

\section{Pueraria lobata (Willd) ohwi (Leguminosae)}

Also known as Kudzu Root, its effects on hyperlipidemia are investigated in a series of studies. Puerarin, the active component significantly reduced the serum lipid concentration or hepatic cholesterol levels in hyperlipidemia rats, when administrated at oral dosages of $300 \mathrm{mg} / \mathrm{kg} /$ day for 4 weeks or injected intraperitoneally at $50 \mathrm{mg} / \mathrm{kg} /$ day for 30 days (Yan et al., 2006). Furthermore, hepatic lipid metabolism was also enhanced in ovariectomized rats by oral administration of Kudzu root (Wang et al., 2004).

\section{Reynoutria japonica Houtt (Polygonaceae)}

Reynoutria japonica Houtt is a synonym of Polygonum cuspidatum siebold and Zucc. Its dried root and rhizome is known as Huzhang in Chinese medicine. Polydatin and resveratrol are the primary active components. It has been found that oral administration of polydatin at dosages of $25 \mathrm{mg}-$ $150 \mathrm{mg} / \mathrm{kg} /$ day for 15-21 days significantly decreased TC, TG, and LDL-C levels or TC/HDL-C ratio in hyperlipidemic hamsters or rabbits (Du et al., 2009; Xing et al., 2009). Intragastrical administration of another component resveratrol at dosages of $10-70 \mathrm{mg} / \mathrm{kg} /$ day for 6 weeks not only decreased levels of serum lipid but also attenuated diet-induced hepatic steatosis and atherosclerosis index in hyperlipidemic mouse (Xie et al., 2014).

\section{Rheum palmatum Linn (Polygonaceae)}

Rheum palmatum Linn., Rheum tanguticum Maxim. Ex Balf., or Rheum officinale Baill belongs to the family of Poygonaceae. Radix Et. Rhizoma Rhei, also known as Dahuang or rhubarb, is derived from the root and rhizome of Polygonaceae members. The main active component includes rhein, aloe emodin, emodin, chrysophanol, and physcion. There is a series of studies exhibited the lipid-lowering effects in animals or patients. For example, the decoction boiled from rhubarb at 8, 16, and $32 \mathrm{~g} / \mathrm{kg} /$ day for 5 days caused fatty degeneration of the hepatic cell in mice. Administration of the powders of rhubarb, at 5 g/day orally for 24 weeks, decreased serum TG and TC levels in patients with diabetic nephropathy (Gao Q. et al., 2010). Rhein, one of the active components or rhubarb, at an oral dosage of $150 \mathrm{mg} / \mathrm{kg} /$ day for 12 weeks, was proved to 
be lowering serum TG, TC, and LDL-C levels in mice with diabetic nephropathy. Thus the lipid-lowering effect of rhubarb, at least, partially dues to the component rhein. Danthron is another extract of rhubarb. It was observed that danthron dosedependently promoted the phosphorylation of AMPK and acetylCoA carboxylase in both HepG2 and C2C12 cells. Meanwhile, danthron significantly reduced sterol regulatory element-binding protein 1c synthesis and fatty acid synthase gene expressions, contributing to the inhibitory effect on lipid metabolism (Han et al., 2013). However, some side-effects, including vomiting, headache, diarrhea and abdominal pain, are reported by some volunteers.

\section{Salviae miltiorrhizae (Lamiaceae)}

It has been described in Section "Application of Plant Products in Hypertension." It is widely used to treat patients with coronary artery disease in China. Studies have showed decrement in levels of TC, TG and LDL-C when administrated with its aqueous extracts orally (50,100, and $150 \mathrm{mg} / \mathrm{kg} / \mathrm{day}$ for 4 weeks in hyperlipidemic rats) (Ji and Gong, 2008) or intravenous injected with tanshinone IIA, the main component (80 $\mathrm{mg} /$ day for 14 days in patients with diabetes) (Jang et al., 2008).

\section{Scutellaria baicalensis Georgi (Lamiaceae)}

Scutellaria baicalensis, known as Huangqin, is derived from the dried root of Scutellaria baicalensis Georgi. The most effective lipid-lowering component is flavonoid compound. Both stem-leaf total flavonoids administrated at oral dosages (25$150 \mathrm{mg} / \mathrm{kg} /$ day) and $0.05 \%$ radix extract added to the diet significantly reduced the serum TG, TC, and LDL-C levels or increased HDL-C levels (Regulska-Ilow et al., 2004). In addition, treatment with flavonoids from the Huangqin leaves increased the activity of lecithin cholesterol acyltransferase in hyperlipidemia rats (Regulska-Ilow et al., 2004), contributing to the lipid-lowering effect.

\section{Senna obtusifolia Linn. (Fabaceae) or Cassia tora Linn. (Leguminosae)}

The ripe seed of Senna obtusifolia Linn. (Fabaceae) or Cassia tora Linn. (Leguminosae) is Semen Cassia, known as Juemingzi. Anthraquinone glycosides are the active component. It has been found that the extract from Semen Cassia at lower doses (8$15 \mathrm{mg} / \mathrm{kg} /$ day) for 35 days, or at higher doses $(180 \mathrm{mg} / \mathrm{kg} /$ day $)$ for 7 days, significantly decreased TC, TG and LDL-C and increased HDL-C in hyperlipidemic animal models. Similar results were induced by administration with the total anthraquinone from Semen Cassia for 2 months in alcohol treated SD rats (Guo et al., 2014).

\section{The Others}

There are many other herbs which show the hypolipidemic effects and widely used in Chinese medicine. These herbs include Rhizoma (Chuanxiong), Rhizoma Curcumae Longae (Jianghuang), Rhizoma Alismatis (Zexie), Semen Plantaginis (Cheqianzi), and Folium Nelumbinis (Heye). The oral administration of the extracts or the active components exerts the hypolipidemic effects and improving lipid metabolism (Guo et al., 2014). In addition, there are some formulae for dyslipidemia which are applied in clinic basing on syndrome differentiation (China Association of Chinese Medicine, 2008). The formulae are listed in Table 2.

\section{Application of Plant Products in Heart Diseases}

Cardiovascular diseases involving the heart includes angina, myocardial infarction, cardiomyopathy, congenital heart disease, and heart failure, etc. Some of the diseases interact on each other or on other vascular diseases. For example, coronary artery diseases involve atherosclerosis, which may be caused by hypertension, hyperlipidemia, whereas heart failure can arise as consequence of large myocardial infarctions. Thus, herbs applied in treatment of hypertension, atherosclerosis and lowering lipid profiles should contribute to attenuating heart diseases too. Here, we present formulae and the natural plants frequently used in clinic and lab studies in alphabetical order.

\section{Formulae}

There are some formulae which are frequently used to treat heart diseases. Chinese herbal formula Sini Tang/dicoction (Aconitum carmichaelii, Zingiber officinale, Glycyrrhiza uralensis, or/and Cinnamomum cassia) was reported to improve cardiac function (ejection fraction and fractional shortening) after myocardial infarction in rats. With ${ }^{1} \mathrm{H}$ NMR and UHPLC-MS measurement, 21-day application of Sini decoction effectively reversed the urinary metabolic profiles in the myocardial infarction rat model (Tan et al., 2012; Sheu et al., 2014). Another formula consists of Terminalia arjuna, Cactus grandiflorous, Crataegus oxyacantha, and Piper nigrum. Preadministration and postadministration of this herbal mixture restore the levels of biomarker of cardiotoxicity, including cardiac marker enzymes, lipids profile, and antioxidant enzymes (Lin et al., 2011). Rhodiola formulation is claimed to relieve the symptoms of ischemic heart disease and improve the electrocardiography in a number of clinical studies (Yu et al., 2014). A meta analysis including 13 studies $(n=1672)$ demonstrated that the effectiveness of Rhodiola formulations was higher compared to control group (symptoms improvement: OR $=2.40,95 \%$ CI: 1.57-3.66, $P<0.0001$; electrocardiography improvement: $\mathrm{OR}=1.48,95 \% \mathrm{CI}: 1.17-1.87, P<0.01)$. In addition, the ORs of symptomatic and electrocardiography improvement in Rhodiola formulations versus other CHMs, versus routine western medicine, and Rhodiola formulations plus routine western medicine versus routine western medicine were 1.51, $2.64,5.63$, and 1.33, 3.11, 2.27, respectively (Yu et al., 2014). These findings indicate that the formulations have a positive effect on treating ischemic heart disease alone or in combination with routine western medicine. In addition, there are some formulae for angina pectoris or coronary heart disease or heart failure which are based on syndrome differentiation (China Association of Chinese Medicine, 2008) and are listed in Tables 3 and 4 . 
TABLE 2 | Formulae for dyslipidemia based on syndrome differentiation.

\begin{tabular}{|c|c|c|c|c|c|}
\hline $\begin{array}{l}\text { Symptom } \\
\text { analysis }\end{array}$ & $\begin{array}{l}\text { Therapeutic } \\
\text { Principle }\end{array}$ & Formulae & Ingredients & Chinese patent drug & Homo remedy \\
\hline Wen Re Yun Jie & Qing Re Li Shi & Long-Dan-Xie-Gan Tang & $\begin{array}{l}\text { Longdancao, Huangqin, Sheng } \\
\text { Dihuang, Zhizi, Chuanmutong, } \\
\text { Chaihu, Danggui, Cheqianzi, } \\
\text { Zexie }\end{array}$ & Sang Ge Jiang Zhi Wan & $\begin{array}{l}\text { Jue Ming Zi, Zexie, } \\
\text { Shanzha Slice }\end{array}$ \\
\hline Tan Shi Nei Zu & Hua Tan Qi Shi & Wen-Dan Tang & $\begin{array}{l}\text { Chenpi, Banxia, Fuling, Zhishi, } \\
\text { Zhuru, Baishu, Dannanxing }\end{array}$ & $\begin{array}{l}\text { Zhi Ke Qing Capsule, Xue Zhi } \\
\text { Ling Tablet, Yue Jian Cao } \\
\text { Oil-emulsion }\end{array}$ & \\
\hline Tan Yu Jie Zhi & Hua Tan Xing Yu & $\begin{array}{l}\text { Er-Chen-Tang and } \\
\text { Xue-Fu-Zhu-Yu Tang }\end{array}$ & $\begin{array}{l}\text { Chenpi, Baixia, Fuling, Chaihu, } \\
\text { Zhishi, Chi Baishao, Sheng } \\
\text { Dihuang, Danggui, } \\
\text { Chuanqiong, Taoren, Honghua }\end{array}$ & $\begin{array}{l}\text { Shan Zhuang Jiang Zhi Tablet, } \\
\text { Tong Mai Jiang Zhi Tablet }\end{array}$ & \\
\hline Pi Xu Shi Sheng & Jiang Pi Li Shi & Wei-Ling Tang & $\begin{array}{l}\text { Chenpi, Houpu, Zisuye, Fuling, } \\
\text { Cangshu, Zhuling, Cheqianzi, } \\
\text { Zexie }\end{array}$ & $\begin{array}{l}\text { Jian Pi Jiang Zhi Granule, Zhi Bi } \\
\text { Tuo Capsule, Jiao Gu Lan Zong } \\
\text { Gan Tablet }\end{array}$ & \\
\hline Gan Shen Yin Xu & Zi Bu Gan Shen & $\begin{array}{l}\text { Yi-Guan-Jian and } \\
\text { Qi-Ju-Di-Huang Wan }\end{array}$ & $\begin{array}{l}\text { Danggui, Sheng Dihuang, } \\
\text { Gouqizi, Shanyao, Fuling, } \\
\text { Shanzhuyu, Zexie, Mudanpi, } \\
\text { Juhua, Beishashen }\end{array}$ & $\begin{array}{l}\text { Jiang Zhi Ling Tablet, Yu Jin } \\
\text { Fang Capsule, Zhi He Shou Wu } \\
\text { Granule }\end{array}$ & \\
\hline Pi Shen Yang Xu & Bu Shen Jian Pi & $\begin{array}{l}\text { You-Gui-Wan and } \\
\text { Shen-Ling-Bai-Shu San }\end{array}$ & $\begin{array}{l}\text { Shu Dihuang, Gouqizi, } \\
\text { Duzhong, Tusizi, Fuzi, Rougui, } \\
\text { Shanyao, Baibiandou, } \\
\text { Dangshen, Fuling, Baishu }\end{array}$ & Dan Tian Jiang Zhi Wan & \\
\hline
\end{tabular}

Formulae should be modified according to specific syndromes and clinical manifestations.

TABLE 3 | Formulae for coronary heart disease and angina pectoris based on syndrome differentiation.

\begin{tabular}{|c|c|c|c|c|c|}
\hline Symptom analysis & Therapeutic Principle & Formulae & Ingredients & Chinese patent drug & Homo remedy \\
\hline Xin Xue Yu Zu & $\begin{array}{l}\text { Huo Xue Hua Yu, Tong } \\
\text { Luo Zhi Tong }\end{array}$ & $\begin{array}{l}\text { Dan-Shen Yin or } \\
\text { Tao-Hong-Si-Wu Tang }\end{array}$ & $\begin{array}{l}\text { Taoren, Danshen, Chuanqiong, } \\
\text { Chishao, Honghua, Sheng } \\
\text { Dihuang, Sharen, Tanxiang }\end{array}$ & $\begin{array}{l}\text { Fu Fang Dan Shen Tablet, } \\
\text { Yin Xing Ye Capsule }\end{array}$ & $\begin{array}{l}\text { Ren Shen San Qi San, } \\
\text { Huo Xue Xin Tong San }\end{array}$ \\
\hline Tan Zhuo Bi Zu & $\begin{array}{l}\text { Hua Tan Xie Zhuo, Yi Bi } \\
\text { Tong Yang }\end{array}$ & $\begin{array}{l}\text { Guo-Lou-Xie-Bai-Ban-Xia } \\
\text { Tang }\end{array}$ & $\begin{array}{l}\text { Guolou, Xiebai, Fabanxia, } \\
\text { Fuling, Chenpi, Zhishi, Xingren }\end{array}$ & Er Chen Wan & \\
\hline Yin Han Ning Zhi & $\begin{array}{l}\text { Wen Tong Xin Yang, Kai } \\
\text { Bi San Jie }\end{array}$ & $\begin{array}{l}\text { Guo-Lou-Xie-Bai-Gui-Zhi } \\
\text { Tang }\end{array}$ & $\begin{array}{l}\text { Guolou, Xiebai, Guizhi, Baijiu, } \\
\text { Biba, Gaoliangjiang }\end{array}$ & $\begin{array}{l}\text { Guan Xin Su He Wan, Su } \\
\text { He Xiang Wan }\end{array}$ & \\
\hline Qi Yin Liang Xu & $\begin{array}{l}\text { Yi Qi Yang Yin, Tong } \\
\text { Luo Zhi Tong }\end{array}$ & Sheng-Mai-San & $\begin{array}{l}\text { Huangqi, Dangshen, Maidong, } \\
\text { Wuweizi, Danshen, Honghua, } \\
\text { Sanqifen }\end{array}$ & $\begin{array}{l}\text { Sheng Mai Yin, Tian Wang } \\
\text { Bu Xin Dan, Bu Xin Qi and } \\
\text { Yang Xin Yin Oral Solution }\end{array}$ & \\
\hline Xin Shen Yang Xu & Wen Bu Xin Shen & Jin-Kui-Shen-Qi Wan & $\begin{array}{l}\text { Guizhi, Danfupian, Sheng } \\
\text { Dihuang, Shanzhuyu, Mudanpi, } \\
\text { Fuling, Zexie, Sanqifen }\end{array}$ & $\begin{array}{l}\text { Jin Kui Shen Qi Wan, Gui } \\
\text { Fu Li Zhong Wan }\end{array}$ & \\
\hline
\end{tabular}

Formulae should be modified according to specific syndromes and clinical manifestations.

\section{Astragalus propinquus (Leguminosae, Fabaceae)}

Injection of Astragalus, also known as Huangqi, is one of the most commonly used Chinese patent medicines for the treatment of chronic heart failure in China as complementary treatment to recommended Western therapies. $\mathrm{Fu}$ et al. (2011) has reviewed 62 clinic trials from 1205 articles. Although the available studies are not adequate to draw a conclusion on the efficacy of the Huangqi injection, it is observed to enhance myocardial contractility, improve circulation, and protect myocardial cells with modern pharmacological tools. For example, in an in vivo rat model of persistent myocardial ischemia produced by occlusion of the left anterior descending coronary artery, pretreatment of Huangqi extract significantly decreased the myocardial infarct size and the serum levels of lactate dehydrogenase, creatine kinase isoform $\mathrm{MB}$, and cardiac troponin. Additionally, this treatment dramatically improved cardiac function, as assessed by $\mathrm{dP} / \mathrm{dt}$, left ventricular developed pressure, and left ventricular end-diastolic pressure (Ma et al., 2013).

\section{Cecropia pachystachya (Urticaceae)}

Cecropia pachystachya, popularly called ambay, is extensively used in herbal medicine of South America. Cecropia pachystachya Mart growing in a temperate region produced a positive inotropic effect on isolated rat hearts independent of beta-adrenergic effect. In contrast, the inotropic effect was prevented by pretreatment with high potassium media (stimulating Na-K-ATP pump), 
TABLE 4 | Formulae for heart failure based on syndrome differentiation.

\begin{tabular}{|c|c|c|c|c|c|}
\hline $\begin{array}{l}\text { Symptom } \\
\text { analysis }\end{array}$ & $\begin{array}{l}\text { Therapeutic } \\
\text { Principle }\end{array}$ & Formulae & Ingredients & Chinese patent drug & Homo remedy \\
\hline Xin Fei Qi Xu & $\mathrm{Bu}$ Yi Xin Fei & $\begin{array}{l}\text { Bao-Yuan Tang and } \\
\text { Bu-Fei Tang }\end{array}$ & $\begin{array}{l}\text { Dangshen, Shu Dihuang, Ziwan, } \\
\text { Sangbaipi, Rougui, Jiu Gancao, } \\
\text { Wuweizi, Fuling, Huangqi, Suan Zaoren }\end{array}$ & $\begin{array}{l}\text { Bu Xin Qi Oral Solution, } \\
\text { Ren Shen Bao Fei Wan }\end{array}$ & Ting Li Zi, Fu Shou Cao \\
\hline Qi Yin Liang Xu & Yi Qi Yang Yin & Wu-Wei-Zi Tang & $\begin{array}{l}\text { Dangshen, Maidong, Wuweizi, Guizhi, } \\
\text { Baishao, Sheng Dihuang, Ajiao, Jiu } \\
\text { Gancao, Jiu Huangqi }\end{array}$ & Sheng Mai Yin & \\
\hline Qi Xu Xue Yu & Yi Qi Huo Xue Li Shui & $\begin{array}{l}\text { Bu-Yang-Huan-Wu Tang } \\
\text { and Wu-Ling San }\end{array}$ & $\begin{array}{l}\text { Huangqi, Danggui, Chishao, Dilong, } \\
\text { Chuanqiong, Honghua, Taoren, Zexie, } \\
\text { Baishu, Zhuling, Fuling, Guizhi }\end{array}$ & $\begin{array}{l}\text { Xin Tong Oral Solution, } \\
\text { Tong Xin Luo Capsule }\end{array}$ & \\
\hline $\begin{array}{l}\text { Yang Xu Shui } \\
\text { Ting }\end{array}$ & Wen Yang Li Shui & Zhen-Wu Tang & $\begin{array}{l}\text { Fuzi, Ganjiang, Niuqi, Chuanqiong, } \\
\text { Chishao, Zexie, Baishu, Zhuling, Fuling }\end{array}$ & $\begin{array}{l}\text { Bu Shen Kang Le Capsule, } \\
\text { Xin Bao Wan }\end{array}$ & \\
\hline Re Tan Yong Fei & $\begin{array}{l}\text { Qing Fei Hua Tan, Xie } \\
\text { Fei Li Shui }\end{array}$ & Qing-Jin-Hua-Tan Tang & $\begin{array}{l}\text { Huangqin, Zhizi, Sangbaipi, Gualou, } \\
\text { Maidong, Chuanbeifen, Jugeng, } \\
\text { Gancao, Yiyiren, Dongguaren, Fuling, } \\
\text { Yuxingcao }\end{array}$ & $\begin{array}{l}\text { Qing Fei Hua Tan Wan, Xian } \\
\text { Zhu Li Shui }\end{array}$ & \\
\hline Han Tan Zu Fei & Wen Fei Hua Tan & $\begin{array}{l}\text { Xiao-Qing-Long Tang and } \\
\text { Ting-Li-Da-Zao-Xie-Fei } \\
\text { Tang }\end{array}$ & $\begin{array}{l}\text { Mahuang, Fabanxia, Chishao, } \\
\text { Ganjiang, Guizhi, Wuweizi, Xixin, Tinglizi }\end{array}$ & $\begin{array}{l}\text { Fu Fang Ge Qing Tablet, Ke } \\
\text { Chuan Capsule }\end{array}$ & \\
\hline Yin Jie Yang Tuo & Gu Yin Hui Yang Jiu Ni & Shen-Fu-Long-Mu Tang & $\begin{array}{l}\text { Shengshaishen, Fuzi, Ganjiang, } \\
\text { Maidong, Wuweizi, Longgu, Muli }\end{array}$ & $\begin{array}{l}\text { Gu Shen Ding Chuan Wan, } \\
\text { Ren Shen Gu Ben Wan }\end{array}$ & \\
\hline
\end{tabular}

Formulae should be modified according to specific syndromes and clinical manifestations.

indicating an inhibition of the pump by ambay (Consolini et al., 2006).

\section{Crataegus spp. (Rosaceae)}

It has been described in previous sections (hawthorn). In isolated perfused hearts, extracts of hawthorn have been reported to show cardioprotective effects without affecting coronary blood flow (Nasa et al., 1993). Simultaneously, they demonstrated vasodilator actions and positive cardiac inotropic effect (Blesken, 1992). These results were verified in a multicenter, placebocontrolled, double-blind study, in which WS 1442, the special extract of hawthorn, improved cardiac function determined by heart rate product (systolic $\mathrm{BP} \times$ heart rate) in patients with NYHA class II heart failure (Weikl et al., 1996). More studies are needed to investigate the mechanisms of hawthorn to attenuate CVDs.

\section{Olea europaea L. (Oleaceae)}

It has been described in previous sections and the main extract from the olive leaves is oleuropein. The rabbits were subjected to a $30 \mathrm{~min}$ period of regional ischemia of the heart followed by a $3 \mathrm{~h}$ reperfusion. Chronic pretreatment with oleuropein reduced the infarct size compared with the control (Andreadou et al., 2006). In doxorubicin-induced cardiomyopathy, oleuropein effectively improved the impaired cardiac geometry and function evidenced by transthoracic echocardiography (Andreadou et al., 2014). In isolated rat hearts, pretreatment with $20 \mu \mathrm{g} / \mathrm{g}$ oleuropein before ischemia resulted in a significant decrease in creatine kinase and reduced glutathione release in the perfusate. Reflow in ischemic hearts induced oxidized glutathione release and membrane lipid peroxidation, which were prevented by oleuropein. The reported data demonstrated the direct cardioprotective effect of oleuropein in the acute events that follow coronary occlusion, and the nutritional benefit of olive oil in the prevention of coronary heart disease (Weikl et al., 1996).

\section{Panax notoginseng (Araliaceae)}

The root of Panax notoginseng, known as Sanqi, Sanchi or Tianqi in East Asian countries, has been identified over 80 variants according to different substitute patterns. There are five main saponins R1, Rb1, Rg1, Rd, and Re, constituting up to $90 \%$ of the total Panax notoginseng used in the pharmacological experiment. A myriad of studies demonstrate the protective effect of notoginseng in cardiac injury. Firstly, it could significantly improve rats' cardiac function evidenced by left ventricular ejection fractions, left ventricular fractional shortening, left ventricular dimensions at end diastole and left ventricular dimensions at end systole (Chen et al., 2011). Secondly, it could reduce infarct size and serum level of creatine kinase in rat models of myocardial ischemia (Yue et al., 2012; Han et al., 2013). Thirdly, it decreased serum levels of lactate dehydrogenase, cardiac troponin I, malondialdehyde and several cytokines, including TNF-a, IL-1 $\beta$ and C-reactive protein (Han et al., 2013). These findings suggest considerable therapeutic potentials of notoginseng for myocardial infarction. Interestingly, Notoginseng combined with the powdered extract of Carthamus tinctorius, an anti-thrombus herbal medicine, or with Sals, the active ingredient of $C$. tinctorius, showed stronger effects to reduce infarct size than either drug alone (Yue et al., 2012; Han et al., 2013; Liu et al., 2014). Thus, combination medication remedy should be considered for CVDs treatment.

\section{Salviae miltiorrhizae (Lamiaceae)}

In addition to hypertension and hyperlipidemia, the dried root of Salviae miltiorrhizae (Danshen) is widely used in China for the treatment of angina pectoris and acute ischemic stroke. It 
has a range of potentially beneficial effects, including improving microcirculation, causing coronary vasodilatation, suppressing the formation of thromboxane, inhibiting platelet adhesion and aggregation, and protecting against myocardial ischemia. Danshen is widely used either alone or in combination with other herbal ingredients for patients with coronary artery disease or CVDs. An acute myocardial infarction model was induced and both infarct size and echocardiographic response were evaluated at different time after surgery. Both Sal and tanshinone (two hypdrophilic and lipophilic compounds) delayed the development of ischemia by decreasing infarct size and improving systolic function post myocardial infarction (Wang et al., 2011). However, a recent review of randomized controlled trials of Danshen in ischemic disease published in mainland China identified 150 trials from 1998 to 2007, but concluded that the quality of these trials has not improved significantly over recent years and the overall quality is still poor (Yu et al., 2014). Since clinical studies have various methodological problems, further high-quality randomized controlled trials should be performed to assess the efficacy of this herb.

\section{MECHANISMS OF PLANT PRODUCTS IN THE ATTENUATION OF CVDS}

For over 2000 years, CVDs have been treated with numerous herbal remedies. However, these herbal remedies have not been well studied using modern cellular and molecular techniques. Based on the existing investigations into mechanisms of single herbal compounds, a summary of these studies is presented in the following section wherein the compounds are discussed according to their target site of activity.

\section{Vascular Smooth Muscle Cells Inhibiting Expression or Activity of Contractile and Structural Proteins}

The blood vessel tonicity is principally controlled by the contraction and relaxation of VSMCs. Upon stimulation, smooth muscle cells develop a contractile force by using the crossbridge cycling between the contractile proteins actins and myosin initiated by the $\mathrm{Ca}^{2+}$-calmodulin interaction and modulated by many other proteins. Herbal medicine that regulates proteins or molecules in this pathway would modulate vascular contraction or relaxation. Firstly, blocking contractile and structural proteins may be one of the ways which natural plants could facilitate vasodilation and vascular remodeling. It has been shown that Tianma (the tuber of an orchid) enhances acetylcholineinduced vasorelaxation or phenylephrine-induced contraction in aortic rings (Feng et al., 2012). In more details, longterm treatment with small doses of Tianma resulted in a significant decrease of the expression levels of primary contractile protein actin to about half the level of the controls, as well as other cytoplasmic structural/cytoskeletal proteins, like Des, Vcl, Pdlim1 and Map4. Secondly, phosphorylation of myosin light chain protein is another target for herbs to determine vascular smooth muscle contraction. A10 cells, rat smooth muscle cells, were treated with kinds of herbs and it was observed that four herbs, Liu-Wei-Di-Huang-Wan, Jia-WeiXiao-Yao-San, Danshen and Gegen, reduced phosphorylation of myosin light chain, suggesting that these most common herbs may be beneficial for smooth muscle cell contractility (Lin et al., 2015).

\section{Regulating Expression of Extracellular Matrix Proteins}

Extracellular matrix is another target involved in vascular tone regulation. ECM proteins such as Postn is specifically induced upon tissue injury and could promote cellular adhesion and movement as well as collagen fibrillogenesis. ECM glycoproteins include Eln, Fbln5 and Prelp, being essential to maintain arterial morphogenesis and vessel elasticity. Tianma could induce upregulation of ECM glycoproteins and down-regulation of ECM proteins in vascular system, thereby regulating blood vessel tonicity by increasing the arterial elasticity and stabilizing the arterial structure (Feng et al., 2012).

\section{Regulating Calcium Levels in PKA/PKG/PKC-Dependent Way}

Calcium levels in VSMCs also contribute to regulating the vascular contraction. Tetramethylpyrazine (TMP, also known as Ligustrazine), is a vasoactive component derived from Ligustium Wollichii Franchat. Using dog mesenteric arterial ring preparations, TMP not only caused a dose-dependent inhibition of vascular contractile responses to $\mathrm{KCl}$ and phenylephrine in solution with calcium, but also inhibited the responses to phenylephrine in $\mathrm{Ca}$-free medium containing $50 \mu \mathrm{M}$ EGTA. These results suggest that herbs may regulate vascular contraction or relaxation by the inhibition of $\mathrm{Ca}^{2+}$ influx, as well as the release of intracellular calcium (Kwan et al., 1990). Consistent with it, baicalein reduced AngII-, vasopressionand endothelin-stimulated increase in $\left[\mathrm{Ca}^{2+}\right]_{i}$ of VSMCs (Saito et al., 1992). This effect was mimicked by a structurally unrelated lipoxygenase inhibitor, 5,8,11-eicosatriynoic acid and was restored by addition of $12(S)$-hydroxyeicosatetraenoic acids (Saito et al., 1992). Thus, baicalein may be representative of the hypotensive herbs mediating $\mathrm{Ca}^{2+}$ levels which may be partly attributed to its inhibition of lipoxygenase and consequent decrease in biosynthesis and release of arachidonic acid-derived vasoconstrictor products.

Alterations in large-conductance $\mathrm{Ca}^{2+}$-activated $\mathrm{K}^{+}$channel $\left(\mathrm{BK}_{\mathrm{Ca}}\right.$ channel) activity play a central role in mediating vasoconstriction and vasodilation. Baicalin $(10-100 \mu \mathrm{M})$ not only concentration-dependently attenuated $\mathrm{KCl}$-contracted mesenteric arteries, but also abolished selective $\mathrm{BK}_{\mathrm{Ca}}$-channel inhibitor IbTX, voltage-dependent $\mathrm{Ca}^{2+}$ channel activator BayK8644, and PKC activator PMA-induced contractions at concentrations of $100 \mu \mathrm{M}$ ( $\mathrm{Lin}$ et al., 2010). These findings suggested that baicalin relaxes mesenteric artery via modulation of the $\mathrm{BK}_{\mathrm{Ca}}$ and $\mathrm{Ca}^{2+}$ channels which may be mediated by the PKC associated pathway. Then, the $\mathrm{BK}_{\mathrm{Ca}}$-mediated vascular contraction is further explored. The cAMP and the cGMP pathways are major regulators of smooth muscle contractility. By cyclic nucleotide assays, baicalin enhanced both 
cAMP and cGMP levels in mesenteric arteries. Based on the theory that smooth muscle relaxants that increase cAMP and cGMP have been shown to activate $\mathrm{BK}_{C a}$ channels through direct phosphorylation of the channel proteins and through elevation of $\mathrm{Ca}^{2+}$ spark frequency, Lin et al. (2010) measured the effects of baicalin on $\mathrm{BK}_{\mathrm{Ca}}$ current by whole-cell patch clamp electrophysiology. Baicalin was observed to enhance $\mathrm{BK}_{\mathrm{Ca}}$ currents in a concentration-dependent manner, which were abolished by combining inhibitors of AC(SQ 22536) and sGC(ODQ), by combining inhibitors of PKA(KT5720) and $\mathrm{PKG}(\mathrm{KT} 5823)$, and PKC activator (PMA) in mesenteric artery smooth muscle cells. These results indicate that baicalininduced mesenteric artery relaxations could be due to $\mathrm{BK}_{C a}$ channel activation occurs not only as a result of PKA and PKG, but also as a result of cross-interaction with PKC (Lin et al., 2010).

\section{Attenuating Proliferation and Migration of VSMCs}

It's well known that stimulation of VSMCs with TNF- $\alpha$ or platelet-derived growth factor-BB induced proliferation. Camellia japonica at concentrations 50, 100, 200, and $400 \mu \mathrm{g} / \mathrm{mL}$ significantly reduced the proliferation rate to $77.85,70.12$, 61.93 , and $56.33 \%$ of the control (TNF- $\alpha$ treated without Camellia japonica) (Park et al., 2015). Similarly, Cinnamon extract inhibited the platelet-derived growth factor-BBinduced proliferation of VSMCs through a G0/G1 arrest, which down-regulated the expression of cell cycle positive regulatory proteins by up-regulating p21 and p27 expression (Kwon et al., 2015). The effects of Camellia japonica on VSMCs migration was evaluated with a wound healing assay. Camellia japonica concentration-dependently suppressed platelet-derived growth factor-BB induced VSMCs wound healing for 24 and $48 \mathrm{~h}$ after injury, indicating the migration inhibiting effects (Park et al., 2015). In addition, baicalein and baicalin induced anti-proliferative and anti-mitogenic effects in VSMCs of rabbit, rat and bovine were well summarized (Huang et al., 2005). These results provide additional evidence for the beneficial cardiovascular effects of natural products.

\section{Anti-inflammation}

Andrographolide is the most active and critical constituent isolated from the leaves of Andrographis paniculata, a herbal medicine widely used as an anti-inflammatory drugs in Asia. The mechanisms of the inflammatory effects of andrographolide in VSMCs were investigated by exposing VSMCs to a proinflammatory stimulus, TNF- $\alpha$. Treating TNF- $\alpha$-stimulated VSMCs with andrographolide suppressed the expression of inducible nitric oxide synthase (NOS), JNK, Akt, and p65 phosphorylation. However, it showed no effect on either IкB $\alpha$ degradation or p38 mitogen-activated protein kinase or ERK1/2 phosphorylation under these conditions. Both treatment with LY294002, a phosphatidylinositol 3-kinase/Akt inhibitor, and treatment with SP600125, a JNK inhibitor, markedly reversed the andrographolide-mediated inhibition of p65 phosphorylation. Thus, andrographolide-mediated inhibition of NF- $\mathrm{KB}$ activity in TNF- $\alpha$-stimulated VSMCs occurs through the JNK-Akt-p65 signaling cascade mechanism, which is independent of ІкB $\alpha$ degradation (Chen et al., 2014). These results collectively suggest that therapeutic interventions using andrographolide can benefit the treatment of vascular inflammatory diseases.

\section{Improving Mitochondrial Function}

In addition to cellular powerhouses, mitochondria are known as critical regulators of cell death. And they are the major cellular source of ROS which causes damage to mitochondrial DNA in human VSMCs in a number of cardiovascular pathologies. Thus, mitochondrial dysfunction may lead to the impairment of various aspects of tissue functioning. It has been known that cultured rat aortic VSMCs treated with AngII for $24 \mathrm{~h}$ exhibited mitochondrial dysfunction, including a decrease in mitochondrial oxygen consumption rates, ATP production and mtDNA levels, as well as the disruption of mitochondrial structural integrity (Lu et al., 2015). Together with the mitochondrial morphological changes, these alterations were reversed by Astragaloside IV, the major active ingredient of Astragalus membranaceus(Fisch.) Bge. (a traditional CHM). Moreover, treatment with Astragaloside IV also reversed the AngII-induced increase in the production of ROS, the increase in NADPH oxidase and xanthine oxidase activity, as well as the decrease in mitochondrial membrane potential and manganeseSOD activity. Furthermore, treatment with Astragaloside IV led to an increase in the mRNA and/or protein levels of peroxisome proliferator-activated receptor-gamma coactivator- $1 \alpha$ (PGC-1 $\alpha$ ), mitochondrial transcription factor A, parkin and dynamin1-like protein1 in the VSMCs. These results indicate that Astragaloside IV exerts beneficial effects on AngII-induced mitochondrial dysfunction in rat VSMCs and that these effects are mediated through the inhibition of ROS overproduction, as well as the promotion of mitochondrial autophagy and mitochondrial biogenesis (Lu et al., 2015).

Overall, herbal medicines discussed here do appear to show pharmacological effects in vitro and in animal studies, which may influence CVDs. In VSMCs, these natural plants exert the protective effect by a series of processes (Figure 1) which include inhibiting expression or activity of contractile and structural proteins, modulating expression of ECM proteins/glycoproteins, regulating calcium levels, attenuating proliferation and migrations, alleviating inflammation, and improving mitochondrial function.

\section{Endothelial Cells \\ Activation of NO Signaling Pathway}

Endothelial cells, which constitute the inner cellular lining of blood vessels, have a key role in regulating vascular homeostasis and function, such as vasorelaxation, vascular integrity, and local inflammation. Endothelium-dependent vasodilation was commonly applied to examine the endothelium function, in which NO is a potent vasodilator and plays an important role in regulating vascular tones. Some herbal medicine functions by targeting at the NO producing process. In studies at the arterial levels, both Lysimachia clethroides and gypenosides elicit vasorelaxation which was abolished by endothelial NOS (eNOS) or guanylyl cyclase inhibitors (Tanner et al., 1999; Lee 


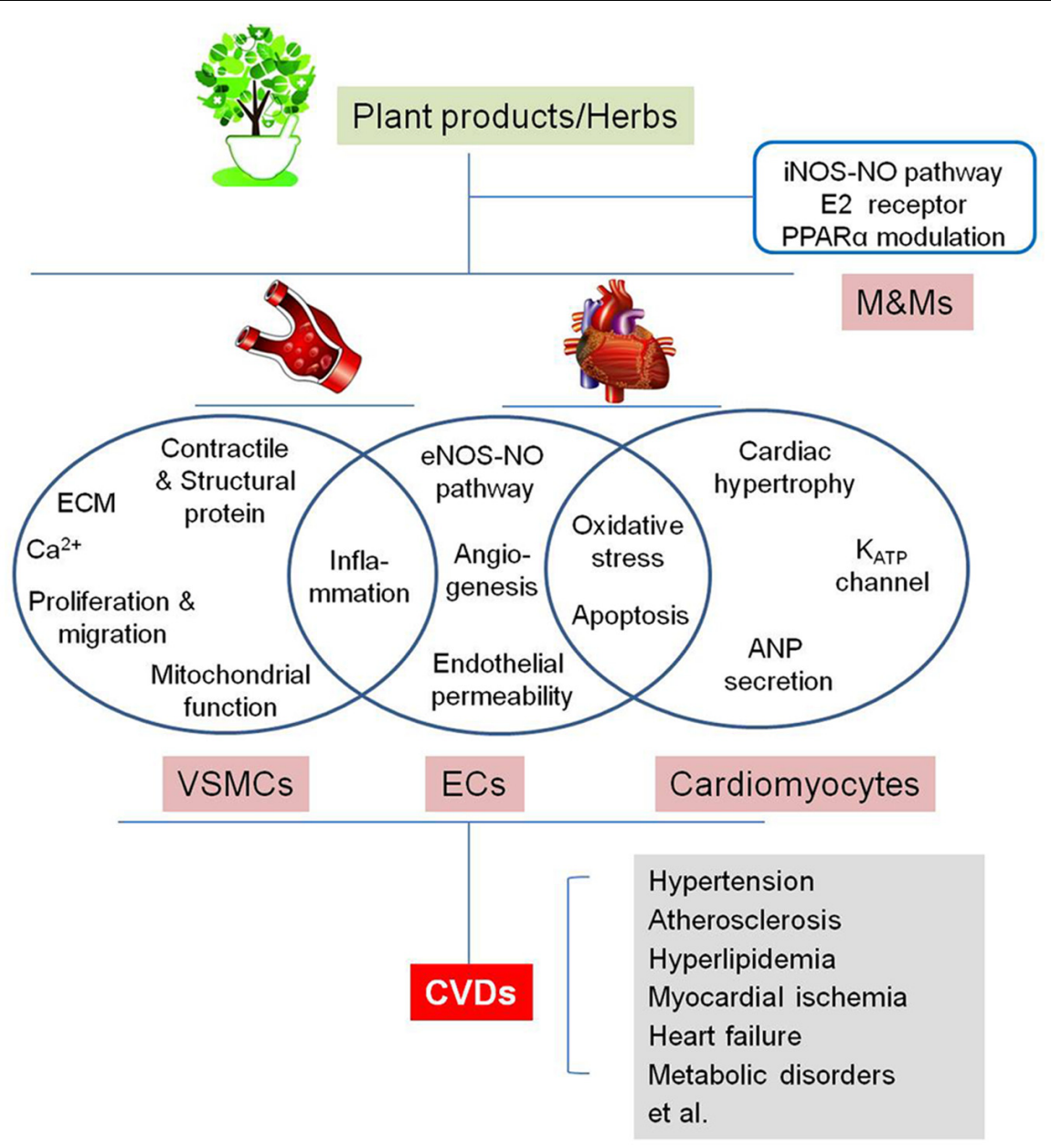

FIGURE 1 | A schematic diagraph indicating the targets on which plant products/herbs act during the pathogenesis of CVDs. Plant products/herbs demonstrate protective effect in CVDs by attenuating damage in cardiomyocytes, endothelial cells (ECs), vascular smooth muscle cells (VSMCs) and macrophages/monocytes (M\&Ms). In VSMCs, plant products/herbs show the beneficial effect by inhibiting expression or activity of contractile and structural proteins, modulating expression of ECM proteins/glycoproteins, regulating calcium levels, attenuating proliferation and migrations, alleviating inflammation, and improving mitochondrial function. In ECs, plant products/herbs show the beneficial effect by inhibition of inflammation, oxidative stress and apoptosis, activation of endothelial nitric oxide synthase(NOS)-NO signaling pathway, induction of angiogenesis and suppression of endothelial permeability. In cardiomyocytes, plant products/herbs show the beneficial effect by inhibiting cardiac hypertrophy, oxidative stress and apoptosis, opening $\mathrm{K}_{\text {ATP }}$ channels and increasing atrial natriuretic peptide (ANP) secretion. In macrophages and monocytes, plant products/herbs show the beneficial effect by inhibition of inducible NOS-NO signaling pathway, activation of estrogen receptor and nuclear receptor peroxisome proliferator activated receptor alpha (PPAR $\alpha$ ). A myriad of molecular, and cellular pathways are favorably modulated by plant products/plants or their extracts.

et al., 2010). Sal B, a hydrophilic caffeic acid derivative of Danshen, exerts an important regulatory function on endothelial tissue of isolated mouse aorta by promoting NO production through the inhibition of arginase activity (Joe et al., 2012). Fo Shou San, an ancient herbal decoction (Chuanxiong Rhizoma and Angelicae Sinensis Radix Danggui in a ratio of 2:3), reversed homocysteine-induced impairment of acetylcholineevoked endothelium-dependent relaxation in rat aortic rings (Bi et al., 2012). In studies at the cellular levels, gypenosides induced a concentration-dependent increase in NO production from cultured bovine endothelial cells (Tanner et al., 1999). Consistent with it, magnesium tanshinoate B (another compound purified from Danshen) or Fo Shou San, also stimulated the release of NO and its metabolites from a human endothelial cell line (ECV304) (O et al., 2000; Bi et al., 2012). By exploring the mechanisms by which NO production is induced, it was observed that the cellular NOS activities were significantly enhanced with a concomitant increase in the levels of constitutive NOS protein mass (O et al., 2000), phosphorylation levels of serine ${ }^{1177}$-eNOS and serine ${ }^{473}$-Akt (Lee et al., 2010; Lu et al., 2010; Bi et al., 2012) by a series of herbal products treatment, including magnesium tanshinoate B, lysimachia clethroides, nordihydroguaiaretic acid (main metabolites of creosote bush) and Fo Shou San. In addition, Fo Shou San elevated intracellular $\mathrm{Ca}^{2+}$ levels and eNOS phosphorylation, which was inhibited by the $\mathrm{Ca}^{2+}$ chelator BAPTA-AM (Bi et al., 2012). These results indicate herbals may benefit endothelial function through increased activity of Akt kinase and eNOS, which is via a rise of intracellular $\mathrm{Ca}^{2+}$. 


\section{Inhibition of Inflammation}

Inflammation plays a crucial role in the pathophysiology of CVDs, in which adhesion molecules, pro-inflammatory cytokines and chemokines are involved. The anti-inflammatory effects of Danshen and its active ingredients (Sal B, Tanshionone IIA, Protoc et al.) were extensively investigated in traditional Chinese medicine for therapy of CVDs. Incubation with TNF $\alpha$ or ADP is widely used to induce inflammation in vitro. In TNF $\alpha$ treated human coronary artery endothelial cells, Sal B significantly inhibited the inflammation and decreased matrix metalloproteinase- 9 expression and activity by blocking the activation of IкB $\alpha / \mathrm{NF} \mathrm{B}$ through JNK and ERK1/2 signaling pathways (Ma et al., 2015). In TNFa treated HUVECs, although neither Sal B nor Tanshinone IIA inhibit the inflammation (Stumpf et al., 2013), Danshen and Protoc attenuated expression and/or release of CD40, VCAM-1 and ICAM-1 as well as cytokines or chemokines (IL-6, IL-8 and MCP-1) (Stumpf et al., 2013). In ADP-treated human platelets, pre-treatment with both Sal B, Tanshionone IIA and Danshen significantly attenuated platelet P-selectin expression (Stumpf et al., 2013). Gypenoside XLIX, a dammarane-type glycoside, is a prominent component of G. pentaphyllum. Studies demonstrate that Gypenoside XLIX inhibits TNF $\alpha$-induced VCAM-1 over expression and hyperactivity in human endothelial cells via a PPAR $\alpha$-dependent pathway (Huang et al., 2007a). These findings provide useful insight into the rational basis of $\mathrm{CHM}$ in the treatment of CVDs.

\section{Attenuation of Oxidative Stress}

It's well known that oxidative stress contributes to endothelium dysfunction. There are a myriad of herbs that demonstrate effects of endothelial cell protection by different mechanisms. For example, Sal B decreases ROS production in the aortic rings. Since ROS easily acts with NO to produce peroxynitrite anions, which is a potent and potentially toxic oxidant that damages various types of biomolecules, it can be deduced that Sal B may exert its protective function by precluding NO consumption through adverse reactions such as peroxynitrite formation and preserving NO bioavailability (Joe et al., 2012). EGb 761 (Ginkgo biloba extract) suppresses oxidative stress in a dose-dependent manner in high glucose-stimulated HUVEC. In addition to inhibition of ROS generation and 8-OHdG content, it attenuated oxidative DNA damage, indicating herbs could exert endothelial protection by alleviating endothelial DNA oxidation (He Y.T. et al., 2014). Echium amoenum extract (a major source of anthocyanins) demonstrated antioxidant and cytoprotective effect in $\mathrm{H}_{2} \mathrm{O}_{2}$-treated HUVECs. In the endothelial injury model, pretreatment of HUVECs with the anthocyanin-rich extract at concentrations of $100-1000 \mu \mathrm{g} / \mathrm{ml}$ reduced the cell death, decreased hydro peroxide concentration, and increased ferric reducing antioxidant power value in both intra- and extra-cellular fluid in a concentration-dependent manner (Safaeian et al., 2015). KRGE reduces the $\mathrm{H}_{2} \mathrm{O}_{2}$-induced cell injury in HUVECs too. The inhibited effect on cell death was blunted by HO-1 inhibitor zinc protoporphyrin. HO-1 is considered to augment the cellular defense against various agents inducing cytotoxic injury. KRGE was observed to induce up-regulation of HO-1 expression in HUVECs, which was abolished by specific silencing of Nuclear factor-eythroid 2related factor 2 (Nrf2, a important anti-oxidant transcription factor) expression (Yang et al., 2011). These results suggest that Korean Red Ginseng may exert a vascular protective effect through Nrf2-mediated HO-1 induction in human endothelial cells. Ophiopogonin D is one of the most bioactive components of Radix Ophiopogon japonicas. Its pretreatment showed a series of antioxidation-related protective effects: (1) reducing $\mathrm{H}_{2} \mathrm{O}_{2}$-induced lipid peroxidation and protein carbonylation, (2) attenuating mitochondrial ROS generation and cell apoptosis, (3) restoring cellular total antiboidative capacity, (4) inhibiting the release of inflammatory cytokines and blocking activation of NFKB and ERK signaling cascades, (5) suppressing the enzymatic activity of catalase, HO-1, and caspases (Qian et al., 2010). In general, these findings support the protective role of herbs as an effective antioxidant in endothelial injury.

\section{Inhibition of Apoptosis}

Although endothelial cell dysfunction occurs in many different disease processes, caspase-dependent apoptotic cell death induced by the extrinsic or intrinsic pathways is identified as a common denominator. Protective effect of KRGE was examined in a serum-deprived apoptosis model, which demonstrated that it could prevent serum deprivation-induced HUVEC apoptosis (Kim et al., 2013). This effect was mediated by increased Bcl-2 and Bcl-XL protein expression, PI3K/Akt-dependent Bad phosphorylation, and eNOS/NO-mediated $S$-nitrosylation of caspases. Cytosolic cytochrome $c$ is known to play a crucial role in the activation of the initiator caspase- 9 and the effector caspase-3. KRGE prevented subcellular redistribution of cytochrome $\mathrm{c}$ from the mitochondrion into the cytosol, resulting in suppression of the extrinsic pathway of apoptosis activation (Kim et al., 2013). The cytoprotective property of KRGE may be valuable for developing new pharmaceutical means that limit endothelial cell death induced during the pathogenesis of vascular diseases.

\section{Inducing Angiogenesis}

Angiogenesis, the formation of new blood vessels from preexisting endothelium, is critical to a variety of processes, both physiologically (embryonic development and wound healing) and pathologically (malignancy and chronic inflammation). It is a complex biologic function comprising several steps, including sequential basement membrane degradation, endothelial cell migration and proliferation, tube formation, inhibition of endothelial proliferation, and the stabilization of new vessels. Modulation of any of these steps would affect new vessel formation. Scutellarin, a known flavone glycoside, is the primary active component of the traditional CHM Erigeron breviscapus (Vant.) Hand. Mazz. It has been shown to induce endothelial cell proliferation and migration, promote capillary-like tube formation, and significantly upregulate platelet endothelial cell adhesion molecule-1 (Gao Z.X. et al., 2010). These results suggest that herbal medicine promotes angiogenesis and may form a basis for angiogenic therapy. 


\section{Suppression of Endothelial Permeability}

Endothelial cell hyperpermeability is another factor implicated in CVDs, in which the importance of tyrosine phosphorylaton and kinase activity in oxidant-mediated loss of cell junction structures has been demonstrated. Extract of salvia miltiorrhiza and its major ingredients, Danshensu and Sal B inhibited TNF $\alpha$-induced endothelial permeability, evidenced by attenuated junctional protein phosphotyrosine and prevention of beta-catenin disassociation from the cytoskeleton (Ding and Yuan, 2007). The mechanism of pharmacological action was further explored. The extract, Danshensu and Sal B also repressed expression of vascular endothelial growth factor and ERK activation in TNF $\alpha$ treated HUVEC cells. In addition, it was found that the extract attenuated the disorganization of vascular endothelial-cadherin, which is likely due to a reduction of vascular endothelial growth factor protein expression as a result of modulation of the ERK signaling pathway (Ding et al., 2005). These findings suggest that active herbal ingredients may help to attenuate CVDs by maintaining the integrity of endothelial junction structure.

In general, herbal medicines do show the beneficial effects in endothelial cells. As shown in Figure 1, these natural plants exert the protective effect by inhibition of inflammation, oxidative stress and apoptosis, activation of eNOS-NO signaling pathway, induction of angiogenesis and suppression of endothelial permeability.

\section{Cardiomyocytes}

\section{Alleviation of Cardiac Hypertrophy}

Pathological cardiac hypertrophy induced by increased sympathetic drive can subsequently lead to congestive heart failure, which represents the major cause of morbidity and mortality worldwide. Astragalus polysaccharide is an active compound extracted from Chinese herb Astragalus membranaceus for "Qi-invigorating." In the in vitro cardiac hypertrophic model induced by isoprenaline, Astragalus polysaccharide treatment inhibited significant increases in cell surface area, total protein content, protein synthesis as well as the expression of hypertrophic markers, including ANP and B-type natriuretic peptide. In addition, Astragalus polysaccharide pretreatment not only alleviated the augmentation of intracellular free calcium during cardiac hypertrophy but also upregulated expression of calcineurin, translocation of nuclear factor of activated $\mathrm{T}$ cells, cytoplasmic 3 into nucleus and activation of calmodulin kinase II (reflected by p-CaMKII) (Dai et al., 2014). According to this research, Astragalus polysaccharide exerted its anti-hypertrophic action via inhibiting $\mathrm{Ca}^{2+}$-mediated calcineurin/NFATc3 and CaMKII signaling cascades, which provided new insights into the application of Astragalus polysaccharide to the therapy of heart diseases.

Chlorogenic acid is an important component of CHM, which acts as an antioxidant scavenging free radicals and preventing inflammation. Pre-treatment of chlorogenic acid in the isoprenaline-induced neonatal rat myocytes, decreased the levels of the hypertrophic markers, ANP, B-type natriuretic peptide and $\beta$-MHC. The level of the intracellular ROS was reduced and the nuclear translocation of NF- $\kappa \mathrm{B}$ was

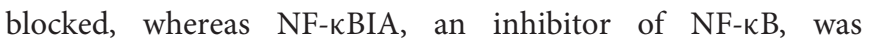
upregulated accordingly. These data reveal that chlorogenic acid may inhibit cardiac hypertrophy by attenuating NF- $\kappa$ B signaling pathway and suppressing ROS production ( $\mathrm{Li}$ et al., 2014).

\section{Attenuation of Oxidative Stress}

Many data have demonstrated that ROS production by myocardial endothelial mitochondria contributes to heart disease and oxidative stress within ventricular myocytes can also be detrimental to the heart. In fact, much of the contractile dysfunction and adverse myocardial remodeling, which has been observed in a wide range of cardiomyopathies, involves oxidative stress and eNOS uncoupling leading to a decrease in NO. There are a series of targets at which herbal medicine act to improve myocardial endothelial function by attenuation of oxidative stress.

(1) Intracellular generation of ROS, including superoxide, hydrogen peroxide and hydroxyl radicals, contributes to the pathogenesis of cellular injury during ischemia and reperfusion in cardiac tissues. Thus, free radical scavenging and inhibition of oxidases will exert protective effect in cardiac injury. Baicalein may provide such therapy by deletion of free radicals, reducing hydroxyl radicals generation by suppressing ironcatalyzed Fenton reaction or decreasing ROS production by inhibiting activities of NADH-oxidase and xanthine oxidase (Huang et al., 2005). (2) ROS may reduce NO bioactivity through the formation of peroxynitrite and NOx. Thus it is easy to understand the oxidized LDLs-induced endothelial dysfunction could be restored by L-arginine and NO. The olive products constitute a rich source of polyphenols such as oleuropein and its derivatives, including hydroxytyrosol, which scavenge free radicals and inhibit the chemical oxidation of LDL. Studies have shown that treatment with high doses of oleuropein at min 1 of reperfusion significantly reduced levels in plasma NOx in hypercholesterolemic rabbits coupled with reduction of infarct size in this group (Andreadou et al., 2014). Therefore, plant products may function as a NO donor to inhibit formation of peroxynitrite and NOx. (3) Circulating or cellular levels of malonaldehyde, TBARS, and protein content in carbonyls serve as Lipid peroxidation and general oxidative stress. The decrease in these biomarkers indicates the protective action of oleuropein, baicalein, crocetin, Allium chinense and Astragalus against oxidative stress by scavenging free radicals (Nasa et al., 1993; Huang et al., 2005; Shen and Qian, 2006; Ren et al., 2010; Andreadou et al., 2014). (4) A great amount of ROS generates from mitochondrial electron transport inhibition, which induces lethal oxidant damage of cardiomyocytes. Qian-Kun-Nin, a $\mathrm{CHM}$ formulation was observed to significantly decreased cell death and attenuate oxidation of DCFH in cells exposed to the mitochondrial site III inhibitor, antimycin A, consistent with a decrease in oxidative stress (Shao et al., 2001). These findings indicate herbal medicine may inhibit oxidative stress by improving mitochondrial function. (5) Myocardial SOD, glutathione peroxidase and myocardial catalase are the 
main antioxidant enzymes, which are dramatically reduced after ischemia and reperfusion. Pretreatment with oleuropein, Astragalus, crocetin or cynaroside kept the SOD activity stable in myocardial infarction models or norepinephrine induced cardiac hypertrophy and therefore left the myocardium in an "antioxidant state"(Nasa et al., 1993; Shen and Qian, 2006; Sun et al., 2011; Andreadou et al., 2014). Thus, keeping the function of antioxidant enzymes standards for another target to inhibit oxidative stress and protect cardiac injury. (6) Nrf2 is a master transcription factor of endogenous antioxidative defense systems. Herbs such as American ginseng could enhance this antioxidant system in rat cardiac H9C2 cells ( $\mathrm{Li}$ et al., 2010). The antioxidant systems include Nrf2 protein expression, Nrf2 nuclear translocation, Nrf2 transcriptional activity, direct $\mathrm{Nrf} 2$ binding to its target gene promoters, and expression of a group of anti-oxidative genes. Thus, herbs may serve as antioxidant enhancer to provide cardioprotection against pathological cardiac injury and remodeling. (7) Doxorubicin triggered impaired left ventricular contractility and inflammatory and degenerative pathology lesions, indicating a successful cardiomyopathy model. Metabonomic analysis revealed treatment of oleuropein induced an favorable variations in the ratio of glycolytic end products (alamine + lactate) to end products of lipid metabolism (acetate), suggesting restoration of the impairment in aerobic glucose metabolism and cardioprotection of ischemia (Andreadou et al., 2014). This may help indentifying novel therapeutic targets in the effect of plant products to protect the heart from injury.

\section{Inhibition of Apoptosis}

$\mathrm{H}_{2} \mathrm{O}_{2}$ treatment induces both oxidative damage and apoptosis, contributing to cardiac injury in cultured rat cardiomyocytes. Cynaroside, a flavonoid compound, has been shown to enhance the endogenous anti-oxidative activity, thereby inhibiting intracellular ROS generation. It also showed the protective effects against $\mathrm{H}_{2} \mathrm{O}_{2}$-induced apoptosis in $\mathrm{H} 9 \mathrm{c} 2$ rat cardiomyoblasts. The mechanism by which cynaroside reduced the apoptotic rate were further explored. Cynaroside pretreatment not only moderated $\mathrm{H}_{2} \mathrm{O}_{2}$-induced disruption of mitochondrial membrane potential, but also increased the expression of anti-apoptotic protein $\mathrm{Bcl}-2$ while decreased the expression of pro-apoptotic protein Bax, and thereby inhibited the release of apoptogenic factors (cytochrome $\mathrm{c}$ and smac/Diablo) from mitochondria in $\mathrm{H} 9 \mathrm{c} 2$ cells. Moreover, cynaroside pretreatment showed an inhibitory effect on the $\mathrm{H}_{2} \mathrm{O}_{2}$-induced increase in JNK and P53 protein expression (Sun et al., 2011). These findings suggest that flavonoid products prevent cardiomyocytes apoptosis in vitro by reducing the endogenous production of ROS, maintaining mitochondrial function, and modulating the JNK and P53 pathways.

\section{Opening $\mathrm{K}_{\text {ATP }}$ Channels}

Cardiac myocyte is the cell types in which $\mathrm{K}_{\mathrm{ATP}}$ channels were originally discovered. It is well established that $\mathrm{K}_{\mathrm{ATP}}$ channels are present at high density in the sarcolemma of cardiac myocytes where they link membrane excitability with the cellular bioenergetic state. The opening of these channels in the heart is believed to mediate ischemic preconditioning, a phenomenon whereby brief periods of ischemia/reperfusion protect the heart against myocardial infarction. Pinacidil is a potassium channel opener which mediate preconditioning at the beginning of sustained hypoxia by opening of sarcolemmal $\mathrm{K}_{\text {ATP }}$ channels (Budas et al., 2004). Similar to it, Guanxinkang treatment benefit the heart function by increasing mRNA and protein expression of $\mathrm{K}_{\text {ATP }}$ subunits (Kir6.1, Kir6.2, SUR2A, SUR2B) in ventricular myocytes (Chen et al., 2010). Keep in line with it, extracts of Astragalus demonstrated its anti-apoptotic effect in $\mathrm{H}_{2} \mathrm{O}_{2}$ injured cardiomyocytes only when the $\mathrm{K}_{\mathrm{ATP}}$ channels were open, whereas the protective effect was not observed when the channels were inhibited by the $\mathrm{K}_{\mathrm{ATP}}$ channel blocker (5-HD, glibenclamide) (Nasa et al., 1993). These findings suggest enhancing the open of $\mathrm{K}_{\mathrm{ATP}}$ channel is one of the important mechanisms by which herbs protect myocardial cells from ischemic injury.

\section{ANP Secretion}

Atrial natriuretic peptide is secreted by the heart atrium cells. ANP binds to a specific set of receptors and acts to reduce the water, sodium and adipose loads on the circulatory system, thereby reducing cardiac output and systemic BP. Emodin, an active anthraquinone constituent isolated from the rhubarb, was observed to increase ANP secretion concomitantly with a decrease in atrial pulse pressure and stroke volume in a concentration-dependent manner. Inhibition of $\mathrm{K}^{+}$channels with tetraethylammonium and glibenclamide or inhibition of L-type $\mathrm{Ca}^{2+}$ channels with nifedipine, attenuated the emodin-induced changes in ANP secretion and atrial dynamics (Zhou et al., 2014). These findings demonstrate that emodin may increase ANP secretion via inhibition of L-type $\mathrm{Ca}^{2+}$ channels through an activation of $\mathrm{K}_{\mathrm{ATP}}$ channel in isolated beating rabbit atria. This study provides a rationale for the use of herbal medicine in the treatment of impairment of the regulation of the cardiovascular homeostasis.

To summarize, plant products/herbal medicines do protective cardiomyocytes from injury. As shown in Figure 1, plant products/herbs show the beneficial effect by inhibiting cardiac hypertrophy, oxidative stress and apoptosis, opening $\mathrm{K}_{\mathrm{ATP}}$ channels and increasing ANP secretion.

\section{Macrophages and Monocytes Inhibition of iNOS-Mediated NO Production}

In the milieu of cardiovascular risk factors that disturb vascular homeostasis, inflammation represents a key early event in vascular pathology, in which monocytes activation, adhesion to the endothelium and infiltration of macrophages into blood vessels are thought to play important pathogenic roles in atherosclerosis and other inflammatory CVDs. It's well known immune cells-derived excessive production of NO by inducible NOS (iNOS) can cause endothelial damage, leading to multiple vascular wall injuries. The transcriptional 
and translational regulation of iNOS in various cell types can be induced by cytokines, growth factors, and endotoxins. Lipopolysaccharide (LPS) stimulation has been shown to enhance iNOS expression, NO production, and macrophage arginase activity in RAW 264.7 macrophages, which were inhibited by Danshen extracts and Sal B. In addition, cytoprotective molecule HO-1 expression was upregulated by these Danshen products too (Joe et al., 2012). SnPP is well characterized as a potent competitive inhibitor of $\mathrm{HO}$ activity. Since the iNOS expression, NO production, and TNF $\alpha$ production, were completely abolished by SnPP, the anti-inflammatory effect of Sal B in macrophages may be related to modulation of HO1. Hb serves as a CO scavenging compound. Preincubation of $\mathrm{Hb}$ for 30 min reversed the inhibitory effect of Sal B on LPSinduced iNOS expression, NO production and NFKB activation in the RAW 264.7, indicating the regulatory effect of Sal B on inflammation may be through mediating the formation of $\mathrm{CO}$ (Joe et al., 2012), which shares the similar mechanism to HO-1 down-regulating proinflammatory cytokines production in LPSstimulated macrophages (Otterbein et al., 2000). Although the mechanism by which HO-1 down-regulates iNOS is incompletely clear, it may involve transcriptional inhibition through CO formation or reduction of heme bioavailability for iNOS synthesis.

\section{Activation of Estrogen Receptor}

Consistent with the effect of Sal B, Tanshinone IIA (another major compound extracted from Danshen) exerts its antiinflammatory effects by inhibition of iNOS gene expression and NO production, as well as inhibition of inflammatory cytokine (IL-1 $\beta$, IL-6 and TNF $\alpha$ ) expression in RAW 264.7 cells (Fan et al., 2009). Since Tan IIA has a similar structure to that of $17 \beta$-estradiol, the estrogenic activities was examined in the immune cells. The Tanshinone IIA's anti-inflammatory effects was mimicked by estradiol and blocked by ICI182187, the antagonist of estrogen receptor (Fan et al., 2009). In fact, the most commonly used alternative herbal medicines for estrogen replacement are soy, black cohosh, dong quai and ginseng. Thus, these herbal medicines may serve as a potential selective estrogen receptor modulator to treat inflammation-associated CVDs without increasing the risk of breast cancer.

\section{Activation of PPAR $\alpha$}

Activation of the nuclear receptor, PPAR $\alpha$, has been demonstrated to modulate many aspects of lipoprotein metabolism and inflammation both in vitro as well as in vivo. The tissue distribution of PPAR $\alpha$ is extensive and it may mediate many of anti-inflammatory and anti-atherogenic effects. Gypenoside XLIX, a dammarane-type glycoside, is one of the prominent components in Gynostemma pentaphyllum. It has been identified as a potent PPAR $\alpha$ activator in HUVEC study (Huang et al., 2007a). Tissue factor is involved in many diseases including CVDs and hence may be an attractive target for directed CVDs therapeutics. In human monocytic THP-1 cells transfected with promoter reporter constructs pTF-LUC, Gyp XLIX (0-300 mM) concentration-dependently inhibited
LPS-stimulated tissue factor promoter activity, mRNA and protein overexpression. This effect was similar to those of Wy-14643, a potent synthetic PPAR $\alpha$ activator, and completely abolished in the presence of the PPAR $\alpha$ selective antagonist MK-886 (Huang et al., 2007b). These data indicate that Gyp XLIX inhibits LPS-induced tissue factor overexpression and enhancement of its activity in human THP-1 monocytic cells via PPAR $\alpha$-dependent pathways, providing new insights into the mechanism of the Chinese herbal plants in CVDs treatment.

Overall, the natural plants/herbs do demonstrate inhibition of inflammation in macrophages and monocytes. These effects are achieved by inhibition of iNOS-NO signaling pathway, which may be through activation of estrogen receptor and nuclear receptor PPAR $\alpha$-dependent signaling pathways (Figure 1).

\section{CONCLUSION}

Although many studies have been missed in this review due to our search strategy and the limited access to some articles, the aforementioned evidence is strongly indicative of the notion that herbs/natural plants are the emerging medicine in the prevention and/or treatment of CVDs. One may predict that herbal remedies will receive even more attention in the coming years. However, some objective limitations should be considered based on the existing literature. Firstly, the CHM are widely studied in China. Accordingly, many articles published on the Chinese literature which may limit these work to be retrieved and public to the world. In addition, a location bias cannot be excluded since trials published in local journals are more likely to report significant results than those published in worldwide mainstream medical journals. Secondly, many of the clinic trials were not well-designed and with poor methodological quality by lacking formal inclusion/exclusion criteria, inadequate description of the randomization procedures, too short duration of therapy and follow-up to achieve conclusive results, et al. Poorly designed and reported clinic trails usually exaggerate the treatment effects which will misled decision making in clinic. Thirdly, compounds contained in one herb, even in an extract of one herb are very complicated. Thus, it is a very tough work to clarify the mechanism of plant products/herb for treating CVDs and interaction with other medicines (including western medicines). Fourthly, many herbal medicine remedies used today have not undergone careful scientific assessment and there still lack preclinical study on the side effects, toxic effects and major drug-to-drug interactions in record. Even though, natural plants/herbs are germs of medicine and should deserve more attention and application. Therefore, to develop new agents with effectiveness and safety from traditional Chinese medicine is a promising way for prevention and treatment of patients with CVDs. However, clinic study criteria should be documented to standardize evaluation of plants/herbs. In addition, international collaboration may be encouraged, promoted and financed from the governments in order to improve the overall research quality. 


\section{AUTHOR CONTRIBUTIONS}

CL drafted the work; YH revised the manuscript. Both CL and $\mathrm{YH}$ contributed substantially to the conception or design of the work, approved the final version to be published, and agreed to be accountable for all aspects of the work in ensuring that questions related to the accuracy or integrity of any part of the work are appropriately investigated and resolved.

\section{REFERENCES}

Acin, S., Navarro, M. A., Arbones-Mainar, J. M., Guillen, N., Sarria, A. J., Carnicer, R., et al. (2006). Hydroxytyrosol administration enhances atherosclerotic lesion development in apo E deficient mice. J. Biochem. 140, 383-391. doi: 10.1093/jb/mvj166

Al Disi, S. S., Anwar, M. A., and Eid, A. H. (2015). Anti-hypertensive herbs and their mechanisms of action: part I. Front. Pharmacol. 6:323. doi: 10.3389/fphar. 2015.00323

Andreadou, I., Iliodromitis, E. K., Mikros, E., Constantinou, M., Agalias, A., Magiatis, P., et al. (2006). The olive constituent oleuropein exhibits antiischemic, antioxidative, and hypolipidemic effects in anesthetized rabbits. J. Nutr. 136, 2213-2219.

Andreadou, I., Mikros, E., Ioannidis, K., Sigala, F., Naka, K., Kostidis, S., et al. (2014). Oleuropein prevents doxorubicin-induced cardiomyopathy interfering with signaling molecules and cardiomyocyte metabolism. J. Mol. Cell. Cardiol. 69, 4-16.

Asgary, S., Naderi, G. H., Sadeghi, M., Kelishadi, R., and Amiri, M. (2004). Antihypertensive effect of Iranian Crataegus curvisepala Lind.: a randomized, double-blind study. Drugs Exp. Clin. Res. 30, 221-225.

Ashraf, R., Aamir, K., Shaikh, A. R., and Ahmed, T. (2005). Effects of garlic on dyslipidemia in patients with type 2 diabetes mellitus. J. Ayub. Med. Coll. Abbottabad 17, 60-64.

Awang, K., Abdullah, N. H., Hadi, A. H., and Fong, Y. S. (2012). Cardiovascular activity of labdane diterpenes from Andrographis paniculata in isolated rat hearts. J. Biomed. Biotechnol. 2012:876458. doi: 10.1155/2012/876458

Bi, C. W., Xu, L., Tian, X. Y., Liu, J., Zheng, K. Y., Lau, C. W., et al. (2012). Fo Shou San, an ancient Chinese herbal decoction, protects endothelial function through increasing endothelial nitric oxide synthase activity. PLOS ONE 7:e51670. doi: 10.1371/journal.pone.0051670

Blesken, R. (1992). [Crataegus in cardiology]. Fortschr. Med. 110, 290-292.

Budas, G. R., Jovanovic, S., Crawford, R. M., and Jovanovic, A. (2004). Hypoxiainduced preconditioning in adult stimulated cardiomyocytes is mediated by the opening and trafficking of sarcolemmal KATP channels. FASEB J. 18, 1046-1048. doi: 10.1096/fj.04-1602fje

Chang, X. X., Yan, H. M., Xu, Q., Xia, M. F., Bian, H., Zhu, T. F., et al. (2012). The effects of berberine on hyperhomocysteinemia and hyperlipidemia in rats fed with a long-term high-fat diet. Lipids Health Dis. 11:86. doi: 10.1186/1476511X-11-86

Chen, F. R., Zhang, N., Liu, P., Zhang, Y. Y., Han, X. H., and Cai, J. F. (2010). [Effects of Guanxinkang on expressions of ATP-sensitive potassium channel subunits Kir6.1, Kir6.2, SUR2A and SUR2B in ischemic myocytes of rats]. Zhong Xi Yi Jie He Xue Bao 8, 458-464.

Chen, S., Liu, J., Liu, X., Fu, Y., Zhang, M., Lin, Q., et al. (2011). Panax notoginseng saponins inhibit ischemia-induced apoptosis by activating PI3K/Akt pathway in cardiomyocytes. J. Ethnopharmacol. 137, 263-270. doi: 10.1016/j.jep.2011. 05.011

Chen, Y., Fu, D. Y., Chen, Y., He, Y. M., Fu, X. D., Xu, Y. Q., et al. (2013). Effects of Chinese herbal medicine Yiqi Huaju Formula on hypertensive patients with metabolic syndrome: a randomized, placebo-controlled trial. J. Integr. Med. 11, 184-194. doi: 10.3736/jintegrmed2013031

Chen, Y. Y., Hsu, M. J., Hsieh, C. Y., Lee, L. W., Chen, Z. C., and Sheu, J. R. (2014). Andrographolide inhibits nuclear factor- $\kappa B$ activation through JNK-Akt-p65 signaling cascade in tumor necrosis factor- $\alpha$-stimulated vascular smooth muscle cells. Sci. World J. 2014:130381.

\section{FUNDING}

This work was supported by National Natural Science Foundation of China 81402646, Zhejiang Provincial Natural Science Fund for Distinguished Young Scholars of China LR17H260001, Hangzhou Science and Technology Plan Project of China 20140633B36 and the Scientific Research Foundation for the Returned Overseas Chinese Scholars, Ministry of Education of China (to CL).

Chen, Z. Y., Su, Y. L., Lau, C. W., Law, W. I., and Huang, Y. (1999). Endotheliumdependent contraction and direct relaxation induced by baicalein in rat mesenteric artery. Eur. J. Pharmacol. 374, 41-47.

China Association of Chinese Medicine (2008). Guidelines for Diagnosis and Treatment of Common Internal Diseases in Chinese Medicine diseases of Modern Medicine. Beijing: Chinese Press of Traditional Chinese Medicine.

Consolini, A. E., Ragone, M. I., Migliori, G. N., Conforti, P., and Volonté, M. G. (2006). Cardiotonic and sedative effects of Cecropia pachystachya Mart. (ambay) on isolated rat hearts and conscious mice. J. Ethnopharmacol. 106, 90-96.

Dai, H., Jia, G., Liu, X., Liu, Z., and Wang, H. (2014). Astragalus polysaccharide inhibits isoprenaline-induced cardiac hypertrophy via suppressing $\mathrm{Ca}(2)(+)$-mediated calcineurin/NFATc3 and CaMKII signaling cascades. Environ. Toxicol. Pharmacol. 38, 263-271. doi: 10.1016/j.etap.2014. 05.008

Ding, M., Ye, T. X., Zhao, G. R., Yuan, Y. J., and Guo, Z. X. (2005). Aqueous extract of Salvia miltiorrhiza attenuates increased endothelial permeability induced by tumor necrosis factor-alpha. Int. Immunopharmacol. 5, 1641-1651.

Ding, M., and Yuan, Y. J. (2007). Study on the mechanisms of an extract of Salvia miltiorrhiza on the regulation of permeability of endothelial cells exposed to tumour necrosis factor-alpha. J. Pharm. Pharmacol. 59, 1027-1033. doi: 10. 1211/jpp.59.7.0016

Du, J., Sun, L. N., Xing, W. W., Huang, B. K., Jia, M., Wu, J. Z., et al. (2009). Lipidlowering effects of polydatin from Polygonum cuspidatum in hyperlipidemic hamsters. Phytomedicine 16, 652-658. doi: 10.1016/j.phymed.2008. 10.001

Fallah Huseini, H., Amini, M., Mohtashami, R., Ghamarchehre, M. E., Sadeqhi, Z., Kianbakht, S., et al. (2013). Blood pressure lowering effect of Nigella sativa L. seed oil in healthy volunteers: a randomized, double-blind, placebo-controlled clinical trial. Phytother. Res. 27, 1849-1853. doi: 10.1002/ptr.4944

Fan, G. W., Gao, X. M., Wang, H., Zhu, Y., Zhang, J., Hu, L. M., et al. (2009). The anti-inflammatory activities of Tanshinone IIA, an active component of TCM, are mediated by estrogen receptor activation and inhibition of iNOS. J. Steroid Biochem. Mol. Biol. 113, 275-280. doi: 10.1016/j.jsbmb.2009.01.011

Fatehi, M., Rashidabady, T., and Fatehi-Hassanabad, Z. (2003). Effects of Crocus sativus petals' extract on rat blood pressure and on responses induced by electrical field stimulation in the rat isolated vas deferens and guinea-pig ileum. J. Ethnopharmacol. 84, 199-203.

Feng, L., Manavalan, A., Mishra, M., Sze, S. K., Hu, J. M., and Heese, K. (2012). Tianma modulates blood vessel tonicity. Open Biochem. J. 6, 56-65. doi: 10. 2174/1874091X01206010056

Fu, S., Zhang, J., Menniti-Ippolito, F., Gao, X., Galeotti, F., Massari, M., et al. (2011). Huangqi injection (a traditional Chinese patent medicine) for chronic heart failure: a systematic review. PLoS ONE 6:e19604. doi: 10.1371/journal.pone. 0019604

Gao, Q., Qin, W. S., Jia, Z. H., Zheng, J. M., Zeng, C. H., Li, L. S., et al. (2010). Rhein improves renal lesion and ameliorates dyslipidemia in $\mathrm{db} / \mathrm{db}$ mice with diabetic nephropathy. Planta Med. 76, 27-33. doi: 10.1055/s-0029-1185948

Gao, Z. X., Huang, D. Y., Li, H. X., Zhang, L. N., Lv, Y. H., Cui, H. D., et al. (2010). Scutellarin promotes in vitro angiogenesis in human umbilical vein endothelial cells. Biochem. Biophys. Res. Commun. 400, 151-156. doi: 10.1016/j.bbrc.2010. 08.034

Gheith, O., Sheashaa, H., Abdelsalam, M., Shoeir, Z., and Sobh, M. (2008). Efficacy and safety of Monascus purpureus Went rice in subjects with secondary hyperlipidemia. Clin. Exp. Nephrol. 12, 189-194. doi: 10.1007/s10157-0080033-x 
Gheith, O., Sheashaa, H., Abdelsalam, M., Shoeir, Z., and Sobh, M. (2009). Efficacy and safety of Monascus purpureus Went rice in children and young adults with secondary hyperlipidemia: a preliminary report. Eur. J. Intern. Med. 20, e57-e61. doi: 10.1016/j.ejim.2008.08.012

Gonzalez-Santiago, M., Martin-Bautista, E., Carrero, J. J., Fonolla, J., Baro, L., Bartolome, M. V., et al. (2006). One-month administration of hydroxytyrosol, a phenolic antioxidant present in olive oil, to hyperlipemic rabbits improves blood lipid profile, antioxidant status and reduces atherosclerosis development. Atherosclerosis 188, 35-42. doi: 10.1016/j.atherosclerosis.2005. 10.022

Guo, M., Liu, Y., Gao, Z. Y., and Shi, D. Z. (2014). Chinese herbal medicine on dyslipidemia: progress and perspective. Evid. Based Complementary Altern. Med. 2014:163036. doi: 10.1155/2014/163036

Han, S. Y., Li, H. X., Ma, X., Zhang, K., Ma, Z. Z., Jiang, Y., et al. (2013). Evaluation of the anti-myocardial ischemia effect of individual and combined extracts of Panax notoginseng and Carthamus tinctorius in rats. J. Ethnopharmacol. 145, 722-727. doi: 10.1016/j.jep.2012.11.036

He, B., Hao, J., Sheng, W., Xiang, Y., Zhang, J., Zhu, H., et al. (2014). Fistular onion stalk extract exhibits anti-atherosclerotic effects in rats. Exp. Ther. Med. 8, 785-792. doi: 10.3892/etm.2014.1790

He, Y. T., Xing, S. S., Gao, L., Wang, J., Xing, Q. C., and Zhang, W. (2014). Ginkgo biloba attenuates oxidative DNA damage of human umbilical vein endothelial cells induced by intermittent high glucose. Pharmazie 69, 203-207.

Herrera-Arellano, A., Miranda-Sanchez, J., Avila-Castro, P., Herrera-Alvarez, S., Jimenez-Ferrer, J. E., Zamilpa, A., et al. (2007). Clinical effects produced by a standardized herbal medicinal product of Hibiscus sabdariffa on patients with hypertension. A randomized, double-blind, lisinopril-controlled clinical trial. Planta Med. 73, 6-12. doi: 10.1055/s-2006-957065

Higashino, S., Sasaki, Y., Giddings, J. C., Hyodo, K., Sakata, S. F., Matsuda, K., et al. (2014). Crocetin, a carotenoid from Gardenia jasminoides Ellis, protects against hypertension and cerebral thrombogenesis in stroke-prone spontaneously hypertensive rats. Phytother. Res. 28, 1315-1319. doi: 10.1002/ptr.5130

Houston, M. C. (2005). Nutraceuticals, vitamins, antioxidants, and minerals in the prevention and treatment of hypertension. Prog. Cardiovasc. Dis. 47, 396-449. doi: $10.1016 /$ j.pcad.2005.01.004

Hu, Y., Ehli, E. A., Kittelsrud, J., Ronan, P. J., Munger, K., Downey, T., et al. (2012). Lipid-lowering effect of berberine in human subjects and rats. Phytomedicine 19, 861-867. doi: 10.1016/j.phymed.2012.05.009

Hu, Y., Sun, B., Liu, K., Yan, M., Zhang, Y., Miao, C., et al. (2016). Icariin attenuates high-cholesterol diet induced atherosclerosis in rats by inhibition of inflammatory response and p38 MAPK signaling pathway. Inflammation 39, 228-236. doi: 10.1007/s10753-015-0242-x

Huang, T. H., Tran, V. H., Roufogalis, B. D., and Li, Y. (2007a). Gypenoside XLIX, a naturally occurring PPAR-alpha activator, inhibits cytokine-induced vascular cell adhesion molecule-1 expression and activity in human endothelial cells. Eur. J. Pharmacol. 565, 158-165. doi: 10.1016/j.ejphar.2007.03.013

Huang, T. H., Tran, V. H., Roufogalis, B. D., and Li, Y. (2007b). Gypenoside XLIX, a naturally occurring gynosaponin, PPAR-alpha dependently inhibits LPS-induced tissue factor expression and activity in human THP-1 monocytic cells. Toxicol. Appl. Pharmacol. 218, 30-36.

Huang, Y., Tsang, S. Y., Yao, X., and Chen, Z. Y. (2005). Biological properties of baicalein in cardiovascular system. Curr. Drug Targets Cardiovasc. Haematol. Disord. 5, 177-184.

Huang, Y., Tsang, S. Y., Yao, X., Lau, C. W., Su, Y. L., and Chen, Z. Y. (2002). Baicalin-induced vascular response in rat mesenteric artery: role of endothelial nitric oxide. Clin. Exp. Pharmacol. Physiol. 29, 721-724.

Huang, Y., Yao, X. Q., Tsang, S. Y., Lau, C. W., and Chen, Z. Y. (2000). Role of endothelium/nitric oxide in vascular response to flavonoids and epicatechin. Acta Pharmacol. Sin. 21, 1119-1124.

Imenshahidi, M., Hosseinzadeh, H., and Javadpour, Y. (2010). Hypotensive effect of aqueous saffron extract (Crocus sativus L.) and its constituents, safranal and crocin, in normotensive and hypertensive rats. Phytother. Res. 24, 990-994. doi: $10.1002 /$ ptr.3044

Imenshahidi, M., Razavi, B. M., Faal, A., Gholampoor, A., Mousavi, S. M., and Hosseinzadeh, H. (2015). The effect of chronic administration of safranal on systolic blood pressure in rats. Iran. J. Pharm. Res. 14, 585-590.

Inuwa, I., Ali, B. H., Al-Lawati, I., Beegam, S., Ziada, A., and Blunden, G. (2012). Long-term ingestion of Hibiscus sabdariffa calyx extract enhances myocardial capillarization in the spontaneously hypertensive rat. Exp. Biol. Med. (Maywood) 237, 563-569. doi: 10.1258/ebm.2012.011357

Jang, E. M., Choi, M. S., Jung, U. J., Kim, M. J., Kim, H. J., Jeon, S. M., et al. (2008). Beneficial effects of curcumin on hyperlipidemia and insulin resistance in high-fat-fed hamsters. Metabolism 57, 1576-1583. doi: 10.1016/j.metabol.2008. 06.014

Jang, S. J., Lim, H. J., and Lim, D. Y. (2011). Inhibitory effects of total ginseng saponin on catecholamine secretion from the perfused adrenal medulla of SHRs. J. Ginseng Res. 35, 176-190. doi: 10.5142/jgr.2011.35.2.176

Ji, W., and Gong, B. Q. (2008). Hypolipidemic activity and mechanism of purified herbal extract of Salvia miltiorrhiza in hyperlipidemic rats. J. Ethnopharmacol. 119, 291-298. doi: 10.1016/j.jep.2008.07.013

Joe, Y., Zheng, M., Kim, H. J., Kim, S., Uddin, M. J., Park, C., et al. (2012). Salvianolic acid B exerts vasoprotective effects through the modulation of heme oxygenase-1 and arginase activities. J. Pharmacol. Exp. Ther. 341, 850-858. doi: 10.1124/jpet.111.190736

Kim, D. Y., Yuan, H. D., Chung, I. K., and Chung, S. H. (2009). Compound $\mathrm{K}$, intestinal metabolite of ginsenoside, attenuates hepatic lipid accumulation via AMPK activation in human hepatoma cells. J. Agric. Food Chem. 57, 1532-1537.

Kim, J. H. (2012). Cardiovascular diseases and Panax ginseng: a review on molecular mechanisms and medical applications. J. Ginseng Res. 36, 16-26. doi: 10.5142/jgr.2012.36.1.16

Kim, Y. M., Kim, J. H., Kwon, H. M., Lee, D. H., Won, M. H., Kwon, Y. G., et al. (2013). Korean Red Ginseng protects endothelial cells from serum-deprived apoptosis by regulating Bcl-2 family protein dynamics and caspase S-nitrosylation. J. Ginseng Res. 37, 413-424. doi: 10.5142/jgr.2013. 37.413

Koyama, T., Taka, A., and Togashi, H. (2009). Effects of a herbal medicine, Hippophae rhamnoides, on cardiovascular functions and coronary microvessels in the spontaneously hypertensive stroke-prone rat. Clin. Hemorheol. Microcirc. 41, 17-26. doi: 10.3233/CH-2009-1148

Kwan, C. Y., Daniel, E. E., and Chen, M. C. (1990). Inhibition of vasoconstriction by tetramethylpyrazine: does it act by blocking the voltage-dependent $\mathrm{Ca}$ channel? J. Cardiovasc. Pharmacol. 15, $157-162$.

Kwon, H., Lee, J. J., Lee, J. H., Cho, W. K., Gu, M. J., Lee, K. J., et al. (2015). Cinnamon and its components suppress vascular smooth muscle cell proliferation by up-regulating cyclin-dependent kinase inhibitors. Am. J. Chin. Med. 43, 621-636. doi: 10.1142/S0192415X1550038X

Lan, J., Zhao, Y., Dong, F., Yan, Z., Zheng, W., Fan, J., et al. (2015). Meta-analysis of the effect and safety of berberine in the treatment of type 2 diabetes mellitus, hyperlipemia and hypertension. J. Ethnopharmacol. 161, 69-81. doi: 10.1016/j. jep.2014.09.049

Lee, J. O., Chang, K., Kim, C. Y., Jung, S. H., Lee, S. W., and Oak, M. H. (2010). Lysimachia clethroides extract promote vascular relaxation via endotheliumdependent mechanism. J. Cardiovasc. Pharmacol. 55, 481-488. doi: 10.1097/ FJC.0b013e3181d7066f

Li, J., Ichikawa, T., Jin, Y., Hofseth, L. J., Nagarkatti, P., Nagarkatti, M., et al. (2010). An essential role of Nrf2 in American ginseng-mediated anti-oxidative actions in cardiomyocytes. J. Ethnopharmacol. 130, 222-230. doi: 10.1016/j.jep.2010. 03.040

Li, L., Zhou, X., Li, N., Sun, M., Lv, J., and Xu, Z. (2015). Herbal drugs against cardiovascular disease: traditional medicine and modern development. Drug Discov. Today 20, 1074-1086. doi: 10.1016/j.drudis.2015.04.009

Li, Y., Shen, D., Tang, X., Li, X., Wo, D., Yan, H., et al. (2014). Chlorogenic acid prevents isoproterenol-induced hypertrophy in neonatal rat myocytes. Toxicol. Lett. 226, 257-263. doi: 10.1016/j.toxlet.2014.02.016

Lin, C. C., Li, T. C., and Lai, M. M. (2005). Efficacy and safety of Monascus purpureus Went rice in subjects with hyperlipidemia. Eur. J. Endocrinol. 153, 679-686. doi: 10.1530/eje.1.02012

Lin, Y., Vermeer, M. A., and Trautwein, E. A. (2011). Triterpenic acids present in hawthorn lower plasma cholesterol by inhibiting intestinal ACAT activity in hamsters. Evid. Based Complementary Altern. Med. 2011:801272. doi: 10.1093/ ecam $/$ nep007

Lin, Y. J., Ho, T. J., Yeh, Y. C., Cheng, C. F., Shiao, Y. T., Wang, C. B., et al. (2015). Chinese herbal medicine treatment improves the overall survival rate of individuals with hypertension among type 2 diabetes patients and modulates 
in vitro smooth muscle cell contractility. PLoS ONE 10:e0145109. doi: 10.1371/ journal.pone.0145109

Lin, Y. L., Dai, Z. K., Lin, R. J., Chu, K. S., Chen, I. J., Wu, J. R., et al. (2010). Baicalin, a flavonoid from Scutellaria baicalensis Georgi, activates large-conductance $\mathrm{Ca} 2+$-activated $\mathrm{K}+$ channels via cyclic nucleotide-dependent protein kinases in mesenteric artery. Phytomedicine 17, 760-770. doi: 10.1016/j.phymed.2010. 01.003

Liu, J., Wang, Y., Qiu, L., Yu, Y., and Wang, C. (2014). Saponins of Panax notoginseng: chemistry, cellular targets and therapeutic opportunities in cardiovascular diseases. Expert Opin. Investig. Drugs 23, 523-539. doi: 10.1517/ 13543784.2014.892582

Lu, J. M., Nurko, J., Weakley, S. M., Jiang, J., Kougias, P., Lin, P. H., et al. (2010). Molecular mechanisms and clinical applications of nordihydroguaiaretic acid (NDGA) and its derivatives: an update. Med. Sci. Monit. 16, RA93-RA100.

Lu, Y., Li, S., Wu, H., Bian, Z., Xu, J., Gu, C., et al. (2015). Beneficial effects of astragaloside IV against angiotensin II-induced mitochondrial dysfunction in rat vascular smooth muscle cells. Int. J. Mol. Med. 36, 1223-1232. doi: 10.3892/ ijmm.2015.2345

Ma, L., Guan, Y. Q., and Du, Z. D. (2015). Salvianolic acid B down-regulates matrix metalloproteinase- 9 activity and expression in tumor necrosis factoralpha-induced human coronary artery endothelial cells. Chin. Med. J. (Engl.) 128, 2658-2663. doi: 10.4103/0366-6999.166037

Ma, X., Zhang, K., Li, H., Han, S., Ma, Z., and Tu, P. (2013). Extracts from Astragalus membranaceus limit myocardial cell death and improve cardiac function in a rat model of myocardial ischemia. J. Ethnopharmacol. 149, 720-728. doi: 10.1016/j.jep.2013.07.036

McKay, D. L., Chen, C. Y., Saltzman, E., and Blumberg, J. B. (2010). Hibiscus sabdariffa L. tea (tisane) lowers blood pressure in prehypertensive and mildly hypertensive adults. J. Nutr. 140, 298-303. doi: 10.3945/jn.109.115097

Modaghegh, M. H., Shahabian, M., Esmaeili, H. A., Rajbai, O., and Hosseinzadeh, H. (2008). Safety evaluation of saffron (Crocus sativus) tablets in healthy volunteers. Phytomedicine 15, 1032-1037. doi: 10.1016/j.phymed.2008.06.003

Moghadam, M. H., Imenshahidi, M., and Mohajeri, S. A. (2013). Antihypertensive effect of celery seed on rat blood pressure in chronic administration. J. Med. Food 16, 558-563. doi: 10.1089/jmf.2012.2664

Mucalo, I., Jovanovski, E., Rahelic, D., Bozikov, V., Romic, Z., and Vuksan, V. (2013). Effect of American ginseng (Panax quinquefolius L.) on arterial stiffness in subjects with type-2 diabetes and concomitant hypertension. J. Ethnopharmacol. 150, 148-153. doi: 10.1016/j.jep.2013.08.015

Nasa, Y., Hashizume, H., Hoque, A. N., and Abiko, Y. (1993). Protective effect of Crataegus extract on the cardiac mechanical dysfunction in isolated perfused working rat heart. Arzneimittelforschung 43, 945-949.

Niu, C., Chen, C., Chen, L., Cheng, K., Yeh, C., and Cheng, J. (2011). Decrease of blood lipids induced by Shan-Zha (fruit of Crataegus pinnatifida) is mainly related to an increase of PPARalpha in liver of mice fed high-fat diet. Horm. Metab. Res. 43, 625-630. doi: 10.1055/s-0031-1283147

O, K., Cheung, F., Sung, F. L., Zhu, D. Y., and Siow, Y. L. (2000). Effect of magnesium tanshinoate $\mathrm{B}$ on the production of nitric oxide in endothelial cells. Mol. Cell. Biochem. 207, 35-39.

Ojeda, D., Jimenez-Ferrer, E., Zamilpa, A., Herrera-Arellano, A., Tortoriello, J., and Alvarez, L. (2010). Inhibition of angiotensin convertin enzyme (ACE) activity by the anthocyanins delphinidin- and cyanidin-3-O-sambubiosides from Hibiscus sabdariffa. J. Ethnopharmacol. 127, 7-10. doi: 10.1016/j.jep.2009. 09.059

Otterbein, L. E., Bach, F. H., Alam, J., Soares, M., Tao Lu, H., Wysk, M., et al. (2000). Carbon monoxide has anti-inflammatory effects involving the mitogenactivated protein kinase pathway. Nat. Med. 6, 422-428.

Park, S. H., Shim, B. S., Yoon, J. S., Lee, H. H., Lee, H. W., Yoo, S. B., et al. (2015). Vascular protective effect of an ethanol extract of Camellia japonica fruit: endothelium-dependent relaxation of coronary artery and reduction of smooth muscle cell migration. Oxid. Med. Cell. Longev. 2015:6309565. doi: $10.1155 / 2016 / 6309565$

Qian, J., Jiang, F., Wang, B., Yu, Y., Zhang, X., Yin, Z., et al. (2010). Ophiopogonin D prevents $\mathrm{H} 2 \mathrm{O} 2$-induced injury in primary human umbilical vein endothelial cells. J. Ethnopharmacol. 128, 438-445. doi: 10.1016/j.jep.2010. 01.031
Regulska-Ilow, B., Biernat, J., Grajeta, H., Ilow, R., and Drzewicka, M. (2004). Influence of bioflavonoids from the radix extract of Scutellaria baicalensis on the level of serum lipids, and the development of laboratory rats fed with fresh and oxidized fats. Nahrung 48, 123-128. doi: 10.1002/food.2003 00382

Reinhart, K. M., Talati, R., White, C. M., and Coleman, C. I. (2009). The impact of garlic on lipid parameters: a systematic review and meta-analysis. Nutr. Res. Rev. 22, 39-48. doi: 10.1017/S0954422409350003

Ren, G., Qiao, H. X., Yang, J., and Zhou, C. X. (2010). Protective effects of steroids from Allium chinense against $\mathrm{H} 2 \mathrm{O} 2$-induced oxidative stress in rat cardiac H9C2 cells. Phytother. Res. 24, 404-409. doi: 10.1002/ptr.2964

Rhee, M. Y., Cho, B., Kim, K. I., Kim, J., Kim, M. K., Lee, E. K., et al. (2014). Blood pressure lowering effect of Korea ginseng derived ginseol K-g1. Am. J. Chin. Med. 42, 605-618. doi: 10.1142/S0192415X14500396

Ried, K. (2016). Garlic lowers blood pressure in hypertensive individuals, regulates serum cholesterol, and stimulates immunity: an updated meta-analysis and review. J. Nutr. 146, 389S-396S. doi: 10.3945/jn.114.202192

Safaeian, L., Haghjoo Javanmard, S., Ghanadian, M., and Seifabadi, S. (2015). Cytoprotective and antioxidant effects of Echium amoenum anthocyanin-rich extract in human endothelial cells (HUVECs). Avicenna J. Phytomed. 5, 157166.

Saito, F., Hori, M. T., Ideguchi, Y., Berger, M., Golub, M., Stern, N., et al. (1992). 12-Lipoxygenase products modulate calcium signals in vascular smooth muscle cells. Hypertension 20, 138-143.

Shao, F., Gu, L., Chen, H., Liu, R., Huang, H., and Ren, G. (2016). Comparation of hypolipidemic and antioxidant effects of aqueous and ethanol extracts of Crataegus pinnatifida fruit in high-fat emulsion-induced hyperlipidemia rats. Pharmacogn. Mag. 12, 64-69. doi: 10.4103/0973-1296. 176049

Shao, Z., Li, C., Becker, L. B., Vanden Hoek, T. L., Schumacker, P. T., Attele, A. S., et al. (2001). Qian-Kun-Nin, a Chinese herbal medicine formulation, attenuates mitochondrial oxidant stress in cardiomyocytes. J. Ethnopharmacol. 74, 63-68.

Shen, X. C., and Qian, Z. Y. (2006). Effects of crocetin on antioxidant enzymatic activities in cardiac hypertrophy induced by norepinephrine in rats. Pharmazie $61,348-352$.

Sheu, J. R., Geraldine, P., and Yen, M. H. (2014). Bioactives and traditional herbal medicine for the treatment of cardiovascular/cerebrovascular diseases. Evid. Based Complementary Altern. Med. 2014:495323. doi: 10.1155/2014/ 495323

Steiner, M., Khan, A. H., Holbert, D., and Lin, R. I. (1996). A double-blind crossover study in moderately hypercholesterolemic men that compared the effect of aged garlic extract and placebo administration on blood lipids. Am. J. Clin. Nutr. 64, $866-870$.

Stumpf, C., Fan, Q., Hintermann, C., Raaz, D., Kurfurst, I., Losert, S., et al. (2013). Anti-inflammatory effects of danshen on human vascular endothelial cells in culture. Am. J. Chin. Med. 41, 1065-1077. doi: 10.1142/S0192415X1350 0729

Sun, X., Sun, G. B., Wang, M., Xiao, J., and Sun, X. B. (2011). Protective effects of cynaroside against $\mathrm{H}(2) \mathrm{O}(2)$-induced apoptosis in $\mathrm{H} 9 \mathrm{c} 2$ cardiomyoblasts. J. Cell. Biochem. 112, 2019-2029. doi: 10.1002/jcb.23121

Tan, G., Liao, W., Dong, X., Yang, G., Zhu, Z., Li, W., et al. (2012). Metabonomic profiles delineate the effect of traditional Chinese medicine sini decoction on myocardial infarction in rats. PLOS ONE 7:e34157. doi: 10.1371/journal.pone. 0034157

Tanner, M. A., Bu, X., Steimle, J. A., and Myers, P. R. (1999). The direct release of nitric oxide by gypenosides derived from the herb Gynostemma pentaphyllum. Nitric Oxide 3, 359-365.

Tassell, M. C., Kingston, R., Gilroy, D., Lehane, M., and Furey, A. (2010). Hawthorn (Crataegus spp.) in the treatment of cardiovascular disease. Pharmacogn. Rev. 4, 32-41. doi: 10.4103/0973-7847.65324

Tsang, S. Y., Chen, Z. Y., Yao, X. Q., and Huang, Y. (2000). Potentiating effects on contractions by purified baicalin and baicalein in the rat mesenteric artery. J. Cardiovasc. Pharmacol. 36, 263-269.

Walden, R., and Tomlinson, B. (2011). "Cardiovascular disease," in Herbal Medicine: Biomolecular and Clinical Aspects, 2nd Edn, eds I. F. F. Benzie and S. Wachtel-Galor (Boca Raton, FL: CRC Press). 
Walker, A. F., Marakis, G., Morris, A. P., and Robinson, P. A. (2002). Promising hypotensive effect of hawthorn extract: a randomized double-blind pilot study of mild, essential hypertension. Phytother. Res. 16, 48-54.

Wang, J. F., Guo, Y. X., Niu, J. Z., Liu, J., Wang, L. Q., and Li, P. H. (2004). Effects of Radix Puerariae flavones on liver lipid metabolism in ovariectomized rats. World J. Gastroenterol. 10, 1967-1970.

Wang, L., Geng, C., Jiang, L., Gong, D., Liu, D., Yoshimura, H., et al. (2008). The anti-atherosclerotic effect of olive leaf extract is related to suppressed inflammatory response in rabbits with experimental atherosclerosis. Eur. J. Nutr. 47, 235-243. doi: 10.1007/s00394-008-0717-8

Wang, X., Wang, Y., Jiang, M., Zhu, Y., Hu, L., Fan, G., et al. (2011). Differential cardioprotective effects of salvianolic acid and tanshinone on acute myocardial infarction are mediated by unique signaling pathways. J. Ethnopharmacol. 135, 662-667.

Weikl, A., Assmus, K. D., Neukum-Schmidt, A., Schmitz, J., Zapfe, G., Noh, H. S., et al. (1996). [Crataegus special extract WS 1442. Assessment of objective effectiveness in patients with heart failure (NYHA II)]. Fortschr. Med. 114, 291-296.

Wong, K. H., Li, G. Q., Li, K. M., Razmovski-Naumovski, V., and Chan, K. (2011). Kudzu root: traditional uses and potential medicinal benefits in diabetes and cardiovascular diseases. J. Ethnopharmacol. 134, 584-607. doi: 10.1016/j.jep. 2011.02.001

Wu, C., Zhang, J., Zhao, Y., Chen, J., and Liu, Y. (2014). Chinese herbal medicine bushen qinggan formula for blood pressure variability and endothelial injury in hypertensive patients: a randomized controlled pilot clinical trial. Evid. Based Complementary Altern. Med. 2014:804171. doi: 10.1155/2014/804171

Xie, H. C., Han, H. P., Chen, Z., and He, J. P. (2014). A study on the effect of resveratrol on lipid metabolism in hyperlipidemic mice. Afr. J. Tradit. Complement. Altern. Med. 11, 209-212.

Xing, W. W., Wu, J. Z., Jia, M., Du, J., Zhang, H., and Qin, L. P. (2009). Effects of polydatin from Polygonum cuspidatum on lipid profile in hyperlipidemic rabbits. Biomed. Pharmacother. 63, 457-462. doi: 10.1016/j.biopha.2008.06.035

Yan, L. P., Chan, S. W., Chan, A. S., Chen, S. L., Ma, X. J., and Xu, H. X. (2006). Puerarin decreases serum total cholesterol and enhances thoracic aorta endothelial nitric oxide synthase expression in diet-induced hypercholesterolemic rats. Life Sci. 79, 324-330. doi: 10.1016/j.lfs.2006.01.016

Yang, H., Lee, S. E., Jeong, S. I., Park, C. S., Jin, Y. H., and Park, Y. S. (2011). Up-regulation of heme oxygenase-1 by Korean red ginseng water extract as a cytoprotective effect in human endothelial cells. J. Ginseng Res. 35, 352-359. doi: 10.5142/jgr.2011.35.3.352

Ye, X. Y., Song, H., and Lu, C. Z. (2008). [Effect of puerarin injection on the mRNA expressions of AT1 and ACE2 in spontaneous hypertension rats]. Zhongguo Zhong Xi Yi Jie He Za Zhi 28, 824-827.
You, Y., Duan, Y., Zhang, X. L., Kang, J., Yan, C. H., Zhang, X. L., et al. (2012). [Effects of astragali radix extract on matrix metalloproteinase 9 expression and atherosclerotic plaque formation in aorta of apolipoprotein E deficient mice fed with high fat diet]. Zhonghua Xin Xue Guan Bing Za Zhi 40, 522-526.

Yu, L., Qin, Y., Wang, Q., Zhang, L., Liu, Y., Wang, T., et al. (2014). The efficacy and safety of Chinese herbal medicine, Rhodiola formulation in treating ischemic heart disease: a systematic review and meta-analysis of randomized controlled trials. Complement. Ther. Med. 22, 814-825. doi: 10.1016/j.ctim.2014. 05.001

Yue, Q. X., Xie, F. B., Song, X. Y., Wu, W. Y., Jiang, B. H., Guan, S. H., et al. (2012). Proteomic studies on protective effects of salvianolic acids, notoginsengnosides and combination of salvianolic acids and notoginsengnosides against cardiac ischemic-reperfusion injury. J. Ethnopharmacol. 141, 659-667. doi: 10.1016/j. jep.2011.08.044

Zhang, C. Y., and Tan, B. K. (1996). Hypotensive activity of aqueous extract of Andrographis paniculata in rats. Clin. Exp. Pharmacol. Physiol. 23, 675-678.

Zhang, J., Liang, R., Wang, L., Yan, R., Hou, R., Gao, S., et al. (2013). Effects of an aqueous extract of Crataegus pinnatifida Bge. var. major N.E.Br. fruit on experimental atherosclerosis in rats. J. Ethnopharmacol. 148, 563-569. doi: 10.1016/j.jep.2013.04.053

Zhou, G. H., Zhang, F., Wang, X. N., Kwon, O. J., Kang, D. G., Lee, H. S., et al. (2014). Emodin accentuates atrial natriuretic peptide secretion in cardiac atria. Eur. J. Pharmacol. 735, 44-51. doi: 10.1016/j.ejphar.2014.04.014

Zhou, J. Y., Zhou, S. W., Zhang, K. B., Tang, J. L., Guang, L. X., Ying, Y., et al. (2008). Chronic effects of berberine on blood, liver glucolipid metabolism and liver PPARs expression in diabetic hyperlipidemic rats. Biol. Pharm. Bull. 31, 1169-1176.

Zhu, F., Huang, B., Hu, C. Y., Jiang, Q. Y., Lu, Z. G., Lu, M., et al. (2005). Effects of total flavonoids of Hippophae rhamnoides L. on intracellular free calcium in cultured vascular smooth muscle cells of spontaneously hypertensive rats and Wistar-Kyoto rats. Chin. J. Integr. Med. 11, 287-292.

Conflict of Interest Statement: The authors declare that the research was conducted in the absence of any commercial or financial relationships that could be construed as a potential conflict of interest.

Copyright (C) 2016 Liu and Huang. This is an open-access article distributed under the terms of the Creative Commons Attribution License (CC BY). The use, distribution or reproduction in other forums is permitted, provided the original author(s) or licensor are credited and that the original publication in this journal is cited, in accordance with accepted academic practice. No use, distribution or reproduction is permitted which does not comply with these terms. 